\title{
Time Triggered Handoff Schemes in Cognitive Radio Networks: A Survey
}

\author{
${ }^{1}$ Usama Mir, ${ }^{2}$ Zeeshan Ahmed Bhatti \\ ${ }^{1}$ Saudi Electronic University (SEU), Saudi Arabia, u.mir@seu.edu.sa \\ ${ }^{2}$ King AbdulAziz University, Saudi Arabia, zbhatti@kau.edu.sa
}

\begin{abstract}
Rapid development in wireless networks has largely raised the demand for spectrum bandwidth. However, current static spectrum allocation policy is unable to meet this ever-growing requirement which causes the spectrum scarcity problem. Cognitive radio network $(\mathrm{CRN})$ has emerged as a grasping solution to scarcity problem where the secondary or unlicensed users are allowed to access the temporary free channels owned by the licensed or primary users. Among other important steps involved in a spectrum management process, handoff plays an essential role since it requires shifting the on-going transmission of a secondary user (SU) to a free channel without degrading the quality of service. An extensive work has been done in the field of spectrum handoff for CRNs. This work is mostly classified in timing, probability, and operating mode based handoff schemes. In this paper, we present a detailed classification and a compressive survey for time triggered handoff schemes. This topic is chosen because in time triggered handoff process, the handoff decision needs to be performed based on continuously sensing the arrival and departure patterns of licensed users, thus making it an important area of research. Therefore, we discuss the pros and cons for time triggered handoff schemes in detail.
\end{abstract}

\section{INTRODUCTION}

With rapid development of wireless networks, the demand for spectrum bandwidth has raised largely [49]. Number of devices utilizing the spectrum (licensed or unlicensed) is growing very fast in contrast to the availability of bandwidth. This spectrum scarcity problem occurred because the current spectrum allocation policy is static which is unable to accommodate the increasing bandwidth demands. In fact, the static allocation policy causes the licensed spectrum bands to be underutilized [36][47].

Cognitive radio network (CRN) comes as an efficient solution to spectrum underutilization [18]. A CRN enables a secondary, an unlicensed or a cognitive radio (CR) user, to utilize the temporarily unoccupied licensed bandwidth of a primary or licensed user in order to enhance the utilization of limited spectrum resources. CR maximizes channel utilization without effecting the well-established spectrum allocation regulation [46].

The main goal of cognitive radio (CR) technology is to allow unlicensed users to opportunistically utilize the spectrum holes (or white spaces) without disrupting communication of primary users (PUs). This opportunistic spectrum usage requires us to develop protocols and algorithms which can adapt to this highly changing environment. Moreover, due to the randomness in PU's behavior with unpredicted arrival and departure timings, it is very difficult to achieve smooth spectrum usage to secondary users (SUs) and limited interference to PUs [110].

Spectrum management process in CRNs usually consists of three different steps: firstly, since CR gets temporary access to available spectrum; therefore, it monitors the available channel and detects the spectrum holes by continuously examining the PU activities known as spectrum sensing [43][48][83]. Next up, there can be multiple SUs accessing specific channels; this access should be coordinated to avoid collisions among users known as spectrum sharing [4][49][95]. The third step is spectrum mobility/handoff [28][46] where an SU should continue its access on a vacant channel in case of arrival of the corresponding PU. Spectrum handoff is an important step in spectrum management process as it requires shifting the on-going transmission of an SU to another free channel without degrading the QoS (quality of service) of licensed users [7].

As defined in [53], the triggering event is considered to be the main cause of handoff initiation. This triggering can be timing, CR user's mobility, probability, or operating mode based. In CR user's mobility based strategy, spectrum handoff can occur due to SUs mobility. This CR movement can be within the same cellular region without changing the current BS (base station) or to another cell, connecting to a new BS [102]. In probability based handoff, the channel prediction probability is chosen to be the main factor for handoff decision [35][120][6] . Thus, algorithms in these types of schemes are designed to predict the probability of future channel being idle or busy. With probabilistic 
estimates, sensing results are also used to make handoff decisions [46][120]. Next is the operating mode based handoff process [61][97]. As mentioned in [53], this handoff is divided in non-hopping and hopping categories. In the former, an SU does not perform a handoff in case of PU arrival and quietly stays on the current channel. In latter, the arrival of a PU may result in triggering of a handoff process and the affected SU may move to another channel. However, an SU may also decide not to perform handoff and stay idle on current channel. In timing based handoff schemes, both the effects of sensing decision and PU arrival rate are considered to trigger a handoff process. This type of handoff requires the movement patterns of PUs to be sensed and monitored carefully since the channel selection and handoff processes are to be performed based on the timing events triggered by a PU entering or leaving a channel. As per our knowledge and highlighted in [53], time triggered handoff is very important in CR networks because it is based on the timing of spectrum sensing [121] and actual handoff process. Time triggered handoff can further be divided in four types (depending on sensing and handoff triggering time) such as non-handoff, proactive handoff, reactive handoff and hybrid handoff/adaptive handoff, respectively [53]. An extensive work has been done in recent literature in field of spectrum handoff in CRNs. To the best of our knowledge, at present, there is no detailed classification and comprehensive survey dealing with time triggered handoff schemes.

Table 1. List of Acronyms used throughout the paper

\begin{tabular}{|l|l|}
\hline \multicolumn{1}{|c|}{ Acronym/Abbreviation } & \\
\hline AHP & Analytical Hierarchy Process \\
\hline BS & Base Station \\
\hline CCC & Common Control Channel \\
\hline CDMA & Code Division Multiple Access \\
\hline CRN & Cognitive Radio Network \\
\hline CTMC & Continuous Time Markov Chain \\
\hline CUWBIN & Cognitive Ultra-Wide Band Industrial Network \\
\hline DFHC & Dynamic Frequency Hopping Communities \\
\hline DSA & Dynamic Spectrum Access \\
\hline FAHP & Fuzzy Analytical Hierarchy Process \\
\hline FCC & Federal Communications Commission \\
\hline FLB & Fuzzy Logic Based \\
\hline FLC & Fuzzy Logic Controller \\
\hline HMM & Hidden Markov Model \\
\hline ISM & Industrial Scientific Medical \\
\hline LTE & Long Term Evolution \\
\hline MAC & Medium Access Control \\
\hline MOTCSD & Modified Optimal Target Channel Sequence Design \\
\hline NPRP & Non-Preemptive Resume Priority \\
\hline PRP & Preemptive Resume Priority \\
\hline PU & Primary User \\
\hline QoS & Quality of Service \\
\hline RF & Radio Frequency \\
\hline SA & Spectrum Aggregation \\
\hline SHCP & Spectrum Handoff based on Commutative Probability \\
\hline SNR & Signal Noise Ratio \\
\hline STBC & Short Time Backup Channel \\
\hline SU & Secondary User \\
\hline UWB & Ultra Wide Band \\
\hline VoD & Video on Demand \\
\hline WLAN & Wireless Local Area Network \\
\hline WRAN & Wireless Regional Area Network \\
\hline & \\
\hline
\end{tabular}


Moreover, we strongly believe that a handoff strategy should be developed by keeping in view the movements of PUs as an important design factor [66][103]. Therefore, in this paper, we address the aforementioned in detail.

The main contributions of our paper are as follows:

- Spectrum handoff strategies based on time triggering are discussed individually in terms of features and limitations.

- Various figures and tables are drawn to present a comparative analysis.

- We present different performance criteria which are important in designing an efficient handoff strategy. We also highlight important papers addressing each of the mentioned criteria.

A list of all acronyms/abbreviations with their full form is provided in Table 1.

The rest of the paper is organized as follows. In the following section, we give a general overview of cognitive radio technology and the handoff process. Existing surveys addressing spectrum handoff process are summarized in Section 3. Time triggered handoff schemes are discussed in section 4. In section 5, important criteria for handoff strategies are detailed. Section 6 highlights the current and future research issues and challenges for time triggered and other handoff schemes in general. Our work is concluded in section 7.

\section{OVERVIEW OF COGNITIVE RADIO AND HANDOFF PROCESS}

\section{A. Cognitive Radio: Basic Concept and Importance}

Currently, spectrum in wireless networks is governed by government agencies through a static assignment policy. Spectrum is assigned to licensed users usually for a longer period of time in large geographical areas. Spectrum is fully utilized in certain portions while a sufficient amount of licensed spectrum remains underutilized due to recently deployed static spectrum access policies [36][47][50] . According to FCC (Federal Communication Commission) [36], at some point of a day, up to $85 \%$ of spectrum assigned to a licensed user may remain idle, showing a huge wastage.

As a result of above, modern-day wireless networks are moving from static and centralized control to distributed and autonomous networks [38][3], where the devices may work more dynamically and can opportunistically select the available spectrum by having frequent interactions and information exchanges with their neighboring devices. By autonomous networks, we mean that the control and information are fully distributed and wireless devices have the capabilities of self-organization and adaptability to cope with frequent network changes. Most commonly, the devices are meant to be infrastructure independent and are designed to enable inter-device interactions over single and multihop networks.

These autonomous as well as opportunistic behaviors are now becoming both possible and necessary by the introduction of cognitive radio technology in wireless networks. A cognitive radio (designed to follow a dynamic access policy) comes as an efficient solution to spectrum underutilization issue. Joseph Mitola defined cognitive radio in [50] as "a radio that employs model based reasoning to achieve a specified level of competence in radio-related domains." Generally, a CR (or a secondary user), considered to be an intelligent wireless network component that is aware of its surroundings through its sensing part, may adapt to the present environment by examining the radio frequency (RF) signals and can learn by interacting with its neighbors. Figure 1 shows that a CR is basically aware of its radio environment, having the capabilities of adapting to these surroundings according to the changes it perceives. To adapt, a CR continuously senses or monitors its environment. It contains the knowledge of the priorities, procedures and needs of its users by learning over time and finally can generate the possible solutions in order to facilitate the necessary communications with its neighbors.

As detailed in [51], CR offers a novel way to solve static spectrum utilization problems. A CR senses the radio environment in order to identify those radio spectrum portions that are not in use by the legacy primary (or licensed) users and provides the incentives for making these unused bands available to perform the services required by the user. The unutilized spectrum portions are known as the spectrum holes or white spaces. In [86], Haykin gave his assessment about a spectrum hole as "a band of frequencies assigned to a primary user, but at a particular time and 
specific geographic location, the band is not being utilized by that user." It is obvious that the first priority to using these holes must always stay with the PUs and the CR user can only utilize it in an opportunistic and/or a negotiated manner without causing any interference to the relative PUs. Apart from licensed access, efficient spectrum sharing in unlicensed bands is also an important concern, which can be addressed via CR technology. The key attribute of unlicensed bands is their "openness" where the users can access the spectrum without license/right at any time. Moreover, due to a large amount of traffic in unlicensed bands, users have less incentives to access the free-to-use spectrum [14]. Therefore, various collisions and conflicts can occur between the users, if unlicensed spectrum access is performed without coordination.

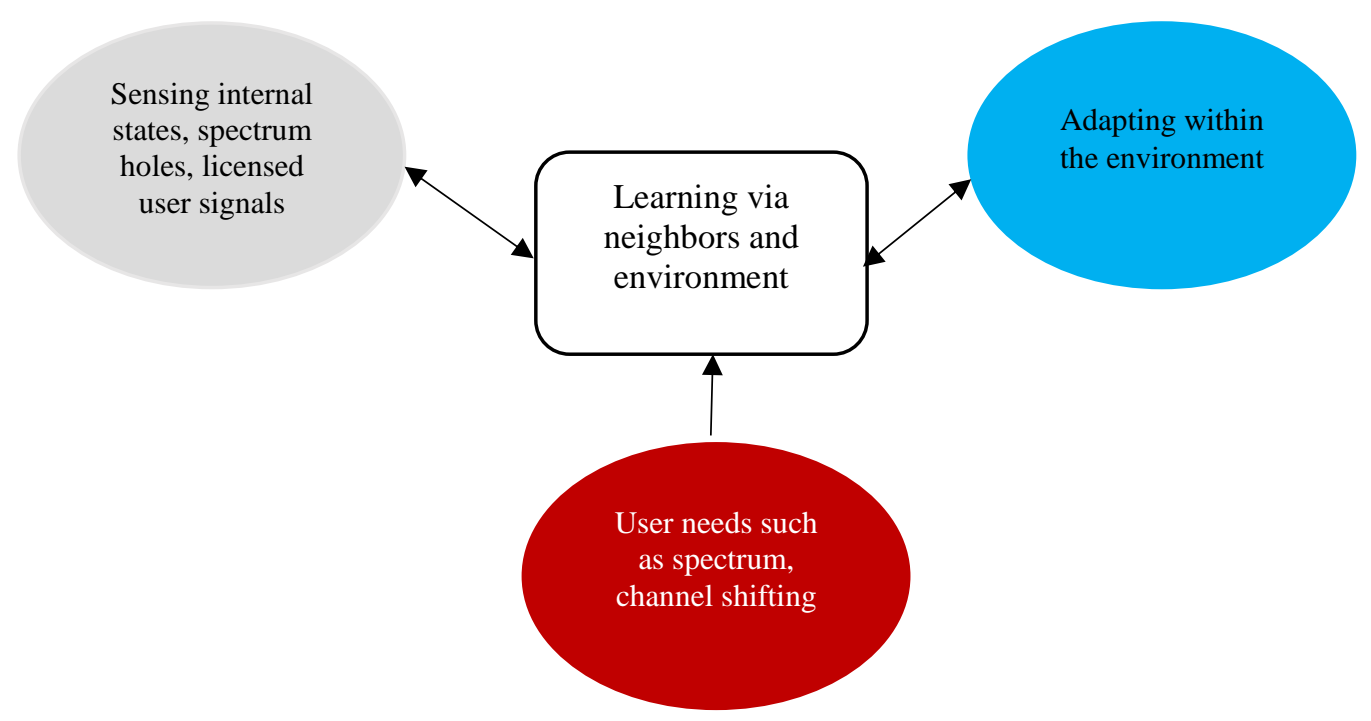

Figure 1. Elementary functions of cognitive radio

Normally, in CRNs channel utilization is maximized without affecting the well-established spectrum allocation regulations [46]. The CR technology enables an SU to determine white spaces opportunistically through spectrum sensing feature. It also helps in detecting the arrival of PU in an SU's operating channel. The best channel is then selected through the spectrum access (sharing) feature and finally, spectrum handoff decision enables an SU to shift its ongoing transmission to another vacant channel on the arrival of a PU. These CR functions are summarized in Table 2 .

Table 2. Brief description of each CR function

\begin{tabular}{|c|c|}
\hline CR FUNCTIONS & DESCRIPTION \\
\hline Sensing & Detection of unused bands or "spectrum holes" by sensing primary users signals \\
\hline Access/Sharing & $\begin{array}{l}\text { Accessing and sharing the available licensed (or unlicensed) spectrum between secondary } \\
\text { users with or without coordination }\end{array}$ \\
\hline Handoff & $\begin{array}{l}\text { Leaving or vacating the licensed spectrum due to the arrival of corresponding licensed } \\
\text { users }\end{array}$ \\
\hline
\end{tabular}




\section{B. Handoff Process in Cognitive Radio Networks}

Unlike traditional wireless networks, in CRNs a handoff should be performed before the arrival and without interrupting the performance of PUs, thus requiring accurate sensing capabilities [93]. Inspired by our previously proposed work in [95], we draw Figure 2 to depict the handoff process in CRNs. Two primary I and J and two secondary $\mathrm{k} 1$ and $\mathrm{k} 2$ users are taken for simplicity. In step 1 (as shown by double arrowed line), $\mathrm{k} 1$ and $\mathrm{k} 2$ are accessing the available band of J. In another instant of time, users k1 and k2 sense the arrival of primary user J (step 2). The two lines with circle heads depict the arrival of J. We assume, in this example, that users $\mathrm{k} 1$ and $\mathrm{k} 2$ have sensed another vacant band of primary user I (step 3), both the users now have to move to spectrum band 2 (step 4) to continue their transmission, thus the spectrum handoff process. This handoff process becomes more complicated with large number of users, thus requiring the development of sophisticated handoff management algorithms.

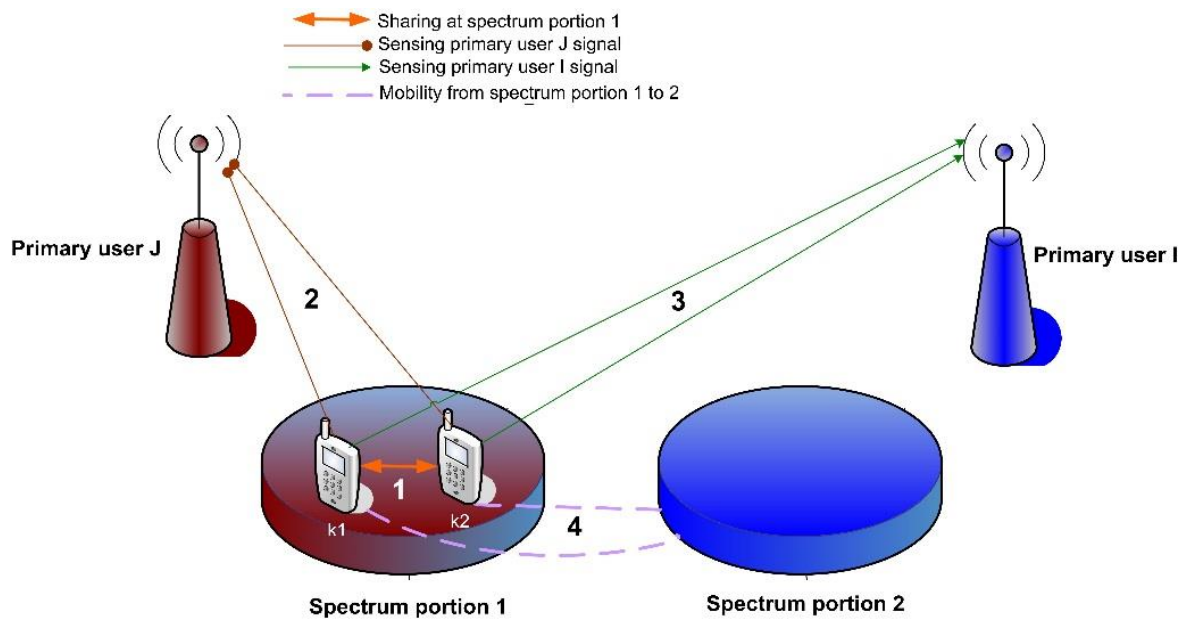

Figure 2. Handoff process in cognitive radio networks [95]

Spectrum handoff in CRNs is generally categorized in two major types as proactive and reactive. In the former, the channel selection is done based on detected traffic patterns of a PU, before the triggering event. In latter, the channel is selected by instant sensing after the occurrence of handoff triggering event. The secondary users affected by the handoff event can resume their transmission on a newly searched channel [30]. Thus, in both types of handoffs, the channel selection is done by continuously monitoring the signals of neighboring PUs. Some authors argue that it is sometimes desirable to have a combination of proactive and reactive handoff strategies to make one hybrid or adaptive solution [20][101][112]. However, in most hybrid solutions, an SU always shifts the channel when a PU arrives. We believe that an SU may have the option of staying silently on a channel while PU is accessing it, this would reduce the number of unwanted handoffs. All these proactive, reactive, and hybrid strategies use time triggering as their essential feature, thus we detail the above mentioned and several other approaches in our forthcoming sections.

\section{RELATED SURVEYS}

Recently, several surveys have been presented addressing the sensing and handoff related issues in CRNs. Table 3 presents a comparative summary of the existing surveys. The works presented in [47][90][52] provide generalized surveys on spectrum mobility in CRNs. The purpose of these surveys is not specifically on time triggered handoff. The authors of [16][34] presented several dynamic spectrum access strategies in CRNs. These strategies are based on auction, game theory, multi agent systems, measurement models, network coded cognitive control channel, fuzzy logic, and Markov chains. Spectrum handoff is also discussed; however the focus remains entirely on spectrum sharing. A few interesting proactive and reactive handoff solutions are also discussed in [65][55], however, the aforementioned do not detail time triggered handoff strategies as discussed in our work. A lot of interesting approaches are missing in [65][42] and the aspect of hybrid solutions is almost ignored. Another short survey is presented in [9] 
which classifies the spectrum handoff strategies for multiple channel CRNs. A classification of spectrum handoff in CRNs is presented in [53], where triggering is discussed briefly however, details are not comprehensive like our work. The authors divided the existing handoff strategies into different classes such as handoff triggered by timing, mobility, probability, and operating mode. They also identified some open research areas for each class including intelligent spectrum handoff, priority based spectrum handoff, spectrum handoff schemes based on green CRNs [25], interference avoidance, spectrum handoff reduction, and optimization of handoff information collection parameters. Several important proactive and reactive schemes were discussed in [53], however, a large amount of recent work remained untouched which we discuss throughout our next sections in detail.

Table 3. Summary of various surveys addressing spectrum handoff/mobility

\begin{tabular}{|l|l|l|l|l|l|l|}
\hline $\begin{array}{c}\text { Reference } \\
\#\end{array}$ & \multicolumn{1}{|c|}{ Topic } & $\begin{array}{c}\text { Handoff } \\
\text { discussion }\end{array}$ & Classification & \multicolumn{1}{|c|}{$\begin{array}{c}\text { Handoff } \\
\text { strategies }\end{array}$} & $\begin{array}{c}\text { Timing based } \\
\text { handoff strategies }\end{array}$ & $\begin{array}{c}\text { Publication } \\
\text { Year }\end{array}$ \\
\hline$[47]$ & Spectrum Management & Yes & No & No & No & 2008 \\
\hline$[52]$ & Mobility Management & Yes & No & No & No & 2010 \\
\hline$[9]$ & Handoff Strategies & Yes & Yes & Yes & No & 2010 \\
\hline$[16]$ & $\begin{array}{l}\text { Dynamic Spectrum } \\
\text { Access }\end{array}$ & Yes & Yes & No & No & 2013 \\
\hline$[34]$ & Spectrum Assignment & Yes & Yes & No & No & 2013 \\
\hline$[42]$ & Handoff Strategies & Yes & Yes & Yes & $\begin{array}{l}\text { No (categorization } \\
\text { based on energy } \\
\text { efficiency) }\end{array}$ & 2013 \\
\hline$[37]$ & $\begin{array}{l}\text { Dynamic Spectrum } \\
\text { Access }\end{array}$ & Yes & Yes & No & No & 2014 \\
\hline$[90]$ & Spectrum Mobility & Yes & No & No & No & 2015 \\
\hline$[65]$ & Mobility Management & Yes & No & Yes & No & 2015 \\
\hline$[55]$ & $\begin{array}{l}\text { Proactive and Reactive } \\
\text { Handoff }\end{array}$ & Yes & No & Yes & Only two & 2016 \\
\hline$[53]$ & Handoff Strategies & Yes & Yes & Yes & $\begin{array}{l}\text { Introduction (Only a } \\
\text { few references related to } \\
\text { time triggering) }\end{array}$ & 2016 \\
& & & & & & \\
\hline
\end{tabular}

\section{TIME TRIGGERED HANDOFF SCHEMES}

Time triggered handoffs are quite relevant to CRNs since the handoff decision totally relates to primary users activities. This requires handoff to be performed by continuously sensing the arrival and departure patterns of PUs thus, making the whole handoff process "time critical". Other handoff schemes consider different factors to perform handoff decision such as CR user mobility, channel probability and operating frequency, however, in time triggered handoff, the actual timing of PU arrival should be considered to trigger a handoff event [53]. Therefore, the future of a CR user's spectrum usage totally depends on how well it senses and monitors the activity of a neighboring PU [118].

According to [53] and as shown in Figure 3, time triggered handoff schemes can be classified in four types such as non-handoff, proactive handoff, reactive handoff, and hybrid handoff strategies, respectively. Recently, a lot of work has been done in the literature to address the time triggered handoff process which is detailed in our forthcoming subsections. However, before going into further discussion about existing time triggered handoff strategies, we depict the behavior of a CR user under time triggered handoff scenario in Figure 4. The process starts with the detection of PU arrival and predicting PU stayed duration on its licensed channel via continuous sensing [45][5]. One possibility for an SU is to predetermine target channels for spectrum handoff, thus keeping a list of channels before transmission. This process is known as Proactive Handoff. A CR user can also decide to wait silently on current channel and allow the PU to finish its transmission and leave the channel. This type of time triggered handoff is called as Non-Handoff. A non-handoff strategy should be designed in a way that a CR user's signal should not affect the transmission of the 
PU since both types of users co-exist on the same channel simultaneously. In another form of time triggered handoff, an SU can leave the channel at the time when the PU arrives. This type of handoff is known as Reactive Handoff. Sometimes it is better to use the combination of both proactive and reactive handoff strategies, thus making a(n) Adaptive/Hybrid strategy. We discuss all important algorithms/strategies related to non-handoff, proactive, reactive and hybrid/adaptive handoff types in the subsequent sections.

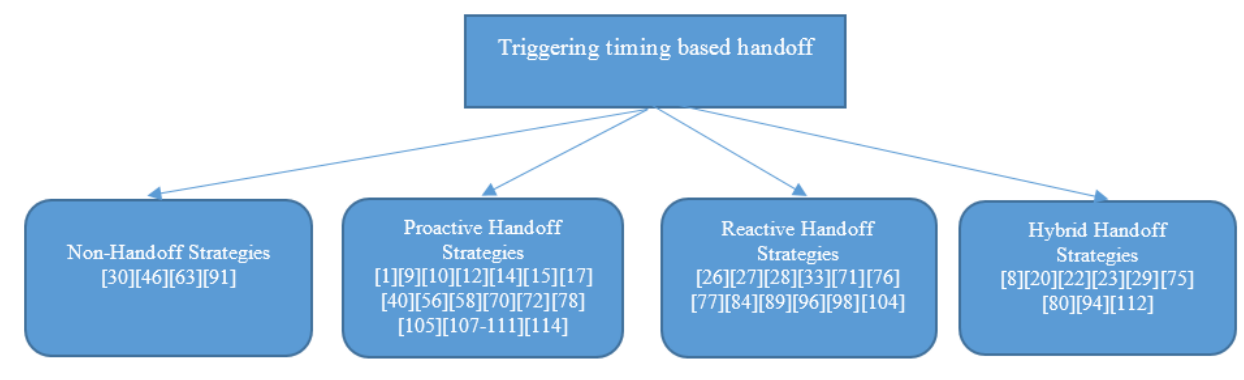

Figure 3. A classification of time triggered handoff strategies

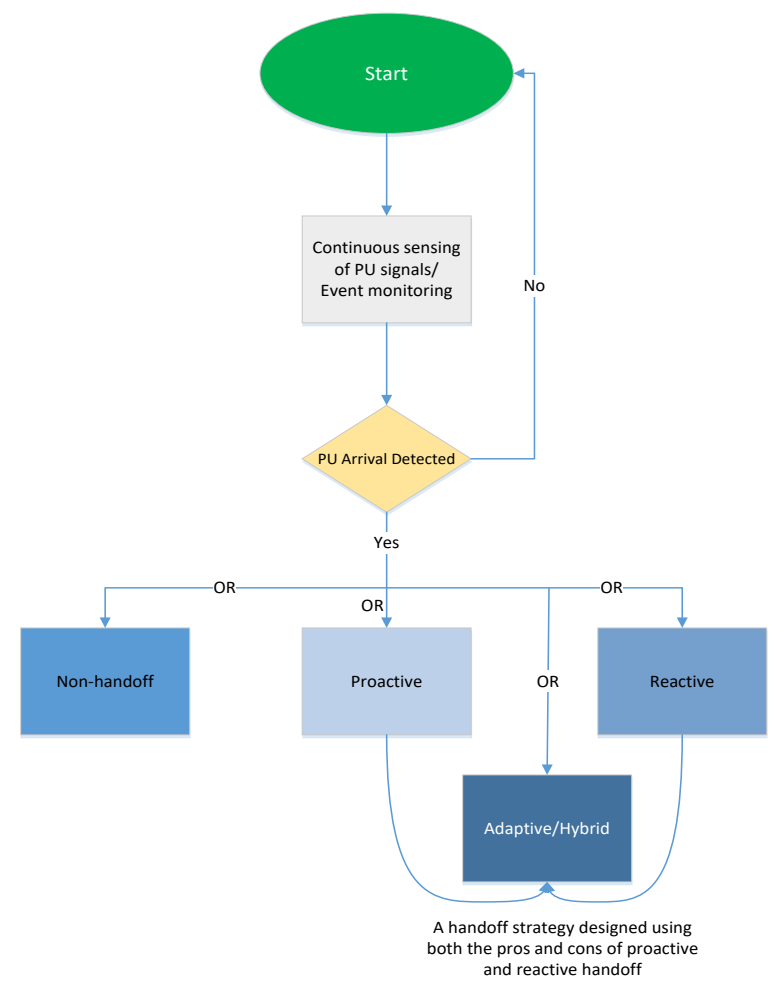

Figure 4. Adaptation of a CR user to different time triggered handoff types based on PU activity

\section{A. Non-handoff Schemes}

In a non-handoff technique shown in Figure 5, on the arrival of a PU, an SU stops its transmission and waits on the current channel " $i$ " for PU to complete its transmission. When PU leaves, the corresponding SU can resume its transmission on channel $i$. The process continues until SU completes transmitting on channel $i$. This handoff technique is similar to the non-hopping approach of IEEE 802.22 [99]. The main feature for a non-handoff algorithm is to have a very low interference with a PU because the data transmission of an SU is fully dependent on PU's activity. Moreover, the cost for searching another vacant channel and performing handoff is eliminated because an SU does 
not shift the channel, however, a non-handoff strategy may suffer from unpredictable waiting latency since a PU can stay on a channel for a pretty long duration.

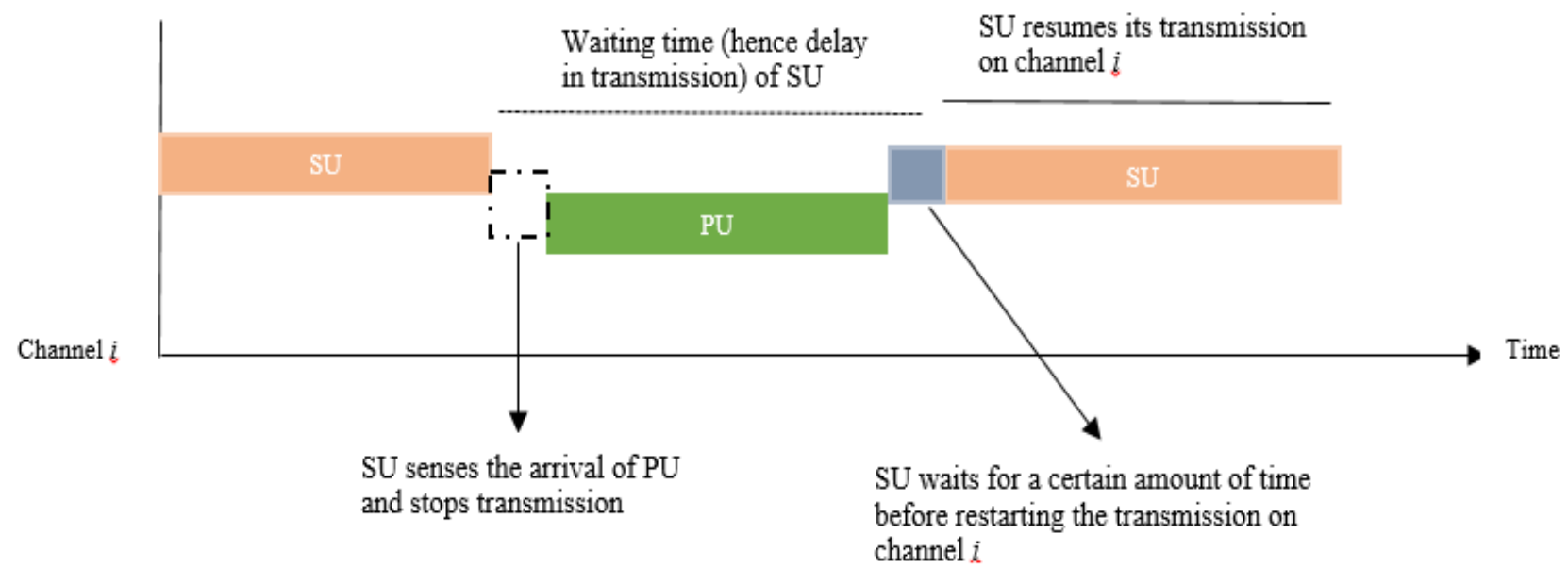

Figure 5. Non-handoff process considering one SU and one PU

The authors of [91][30][63] adapted a non-handoff behavior in their proposed models. Normally, in a nonhandoff process, the average handoff delay for an SU's transmission is the average period a PU occupies a channel. In [91], it is argued that performing handoff is a time consuming process since SUs have to search for other channels and reconfigure their operating parameters accordingly. Thus, authors believe on the idea of avoiding handoffs at PU arrival and staying and waiting silently on a channel unless the corresponding PU finishes its transmission. In the proposed model of [91], SUs having interruption in their transmissions (due to PU arrival) should maintain priority queues where they have to wait unless PU leaves the channel and other uninterrupted SUs finish their transmissions. This waiting time adds an extra delay, however, it reduces users collisions in accessing a channel.

In [30], the authors compared cumulative handoff delay for non-handoff and random selection handoff strategies. These two approaches are renamed as "always staying" and "always changing" strategies, respectively. In an always staying approach, the target channels are predetermined since an SU does not perform a handoff. For an always changing scheme on the other hand, future channel has to be determined randomly on PU arrival (thus, this approach can also be classified under proactive handoff category). Through experiments, it is shown that with higher value of PU arrival, the random selection has more cumulative handoff delay compared to the non-handoff scheme. This delay is usually because the interrupted SUs with random selection method must wait for longer periods when changing their operating channels since the "selected target channels" are likely to be busy. Another important non-handoff strategy is given in [46] which is detailed in the survey presented in [53].

Like above, in [63] load balancing is considered to be the primary goal for channel selection. At first, the optimal number of channels is sensed using CR sensing capability. Later, the best/optimal channel selection is done using probability based analysis. The prime objective of authors is to minimize the overall system time which remains at lower side due to the non-handoff policy adapted in [63].

\section{B. Proactive Handoff Schemes}

In proactive handoff strategies (Figure 6), the future channel for data communication is determined according to the detected traffic patterns of a PU, before the handoff triggering event. Prior to the arrival of a PU, an SU can shift its on-going transmission to another backup channel reducing the transmission delay. There is a lot of work done for proactive handoff in the current literature. For the sake of simplicity and explanation, below we divide the existing literature on proactive handoff in several subsections. In addition, the pros and cons of the proactive approaches are summarized in Table 4. 


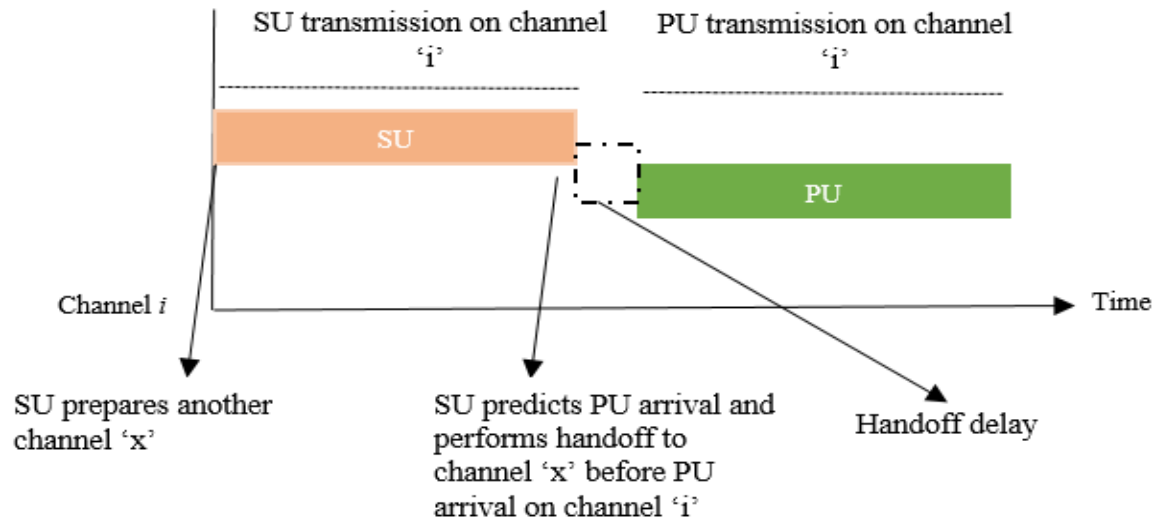

Figure 6. Proactive handoff process at channel 'i' inspired by [46]

Table 4. Summarized list of pros and cons of several proactive handoff schemes

\begin{tabular}{|c|c|c|}
\hline \multicolumn{3}{|c|}{ Pros and Cons of Proactive Handoff Schemes } \\
\hline $\begin{array}{l}\text { Approach/ } \\
\text { (acronym) }\end{array}$ & Advantage & Limitation \\
\hline STBC & $\begin{array}{l}\text { Minimization in handoff delay compared to } \\
\text { approaches with no backup channel }\end{array}$ & Design and algorithmic complexity \\
\hline $\begin{array}{c}\text { Fuzzy logic } \\
\text { based } \\
\text { schemes }\end{array}$ & $\begin{array}{ll}\text { - } & \text { Maximum channel utilization } \\
\text { - } & \text { Thorough comparison with existing } \\
\text { random handoff schemes proves that SUs } \\
\text { get their maximum throughput }\end{array}$ & $\begin{array}{ll}\text { - } & \text { No learning via environment } \\
\text { - } & \text { Wastage of backup channels } \\
\text { - } & \text { No robustness } \\
\text { - } & \text { Design and architectural complexity } \\
\text { - } & \text { Hardware overhead }\end{array}$ \\
\hline CPSH & QoS is high & Wastage of backup channels \\
\hline $\begin{array}{c}\text { Markov } \\
\text { based } \\
\text { approaches }\end{array}$ & $\begin{array}{l}\text { - Accurate sensing using Markov models } \\
\text { - Well defined states of channel usage, idle } \\
\text { transmission and handoff for a user } \\
\text { - SUs can access a channel in the presence } \\
\text { of PUs }\end{array}$ & $\begin{array}{l}\text { - Limited comparison with existing solutions } \\
\text { - In some solutions, despite collision } \\
\text { avoidance, SUs signal can still affect the } \\
\text { transmission of PUs which is unacceptable } \\
\text { - } \quad \begin{array}{l}\text { Only one or two authors model the handoff } \\
\text { process in continuous time }\end{array}\end{array}$ \\
\hline MOTCSD & $\begin{array}{l}\text { Overall processing (sensing and handoff) time is at } \\
\text { minimal level }\end{array}$ & Traditional limitation of SUs acting greedy at times \\
\hline
\end{tabular}

\section{I. $\quad$ Short Time Backup Channel (STBC)}

Short time backup channel (STBC) is a proactive handoff strategy proposed in [9] where the backup channel for SU transmission is selected before the PU arrival. The handoff decision in STBC is dependent on the QoS of current channel. If the current channel meets the transmission (or QoS) requirements of an SU, the backup channel is released. Otherwise, the SU has to shift its transmission to the backup channel. Proposed strategy keeps the backup channel for a short duration so it achieves better bandwidth utilization compared to schemes maintaining a permanent backup channel. A minimum level of handoff delay can be achieved by STBC as compared to approaches with no backup channel because the target channel is selected before the occurrence of handoff triggering event. However, 
maintenance of backup channels results in bandwidth underutilization as well as a high algorithmic complexity since users have to manage current and backup channels simultaneously. Some other proactive schemes based on the idea of "not-using" a common control channel (CCC) for SUs communications are proposed in [110][109], respectively. The information about these schemes are already given in [53] quite comprehensively, therefore we skip the details here. In addition, the authors of [12] analyze the performance of CR handoff management system with and without the inclusion of a common control channel. Through extensive analytical and experimental results, it is revealed that the addition of a CCC leads to an additional delay, however, under ad-hoc network conditions, adding CR nodes is more costly than incorporating a CCC.

\section{II. $\quad$ Fuzzy Logic Based (FLB) Proactive Handoff}

An interesting Fuzzy logic based proactive spectrum handoff scheme is presented in [58]. The main purpose of fuzzy logic is to handle problems more efficiently compared to mathematical models. The proposed algorithm is based on two Fuzzy logic controllers (FLCs). The first controller is there to measure the distance between PUs and SUs. It also estimates the transmission power for an SU so that it should not affect the transmission of neighboring PUs. The second controller is designed to check whether an SU should stay on the current channel or leave. A handoff is initiated, if the QoS for an SU is not up to the mark and thus, the SU is causing considerable amount of interference to the transmission of neighboring PUs. The main advantage of this strategy is the use of Fuzzy logic which provides an efficient estimation of handoff decision parameters such as QoS and transmission power of an SU and its interfering effects on a PU's transmission. Moreover, since no backup channel is used in this approach, the channel utilization remains maximal, however, the use of FLCs makes this approach suffer traditional limitations of having no robustness and overall architectural and design complexities.

A proactive handoff scheme is presented in [78] that applies Fuzzy analytic hierarchy process (FAHP) for the handoff decision. This strategy reserves a number of backup channels in advance with the current operating channel. The pre-determined channels are characterized on the basis of required QoS and continuing validity indicators. The authors present extensive simulations to analyze proposed FAHP on the basis of switching frequency, overall system throughput and system delay. Comparisons with traditional random handoff schemes reflect that FAHP effectively reduces the latency and number of handoffs while improving the overall system throughput, but no limitations are identified in the results. We believe that this approach also suffers with the same drawbacks mentioned above for FLC based proactive approach [58].

\section{Commutative Probability based Spectrum Handoff (CPSH)}

The authors of [10] proposed a proactive handoff strategy that is based on cumulative probability (CPSH). In this technique, a PU decides on whether to stay on its current operating channel or perform handoff depending on the results of probability estimation algorithm. According to this algorithm, an SU performs a handoff when: 1) a PU comes back to its licensed channel currently occupied by an SU; 2) the current channel does not meet the bandwidth requirements for an SU to continue its transmission; and 3) the quality of new channel is better than the current one (in terms of throughput). Another improvement in the proposed model is the use of a backup channel for a short duration (very much similar to [9]). If the current channel meets the transmission requirements for an SU, the backup channel is released to maximize the channel utilization, thus, providing a better QoS and minimum handoff delay for SUs. Though, backup channel is used for short durations, it can still result in channel underutilization since it remains free unless and until an SU initiates transmission on it.

\section{Markov Models for Proactive Spectrum Handoff}

Random appearance of a PU can sufficiently degrade the ongoing communication of an SU on a specific spectrum band. This disruption in transmission can lead to lower throughput in CRNs. To cope with this challenge, the authors of [24] propose a hidden Markov model (HMM) based scheme to optimize handoff decision in CRNs. HMM is used to correct the spectrum sensing sequence in order to enhance the spectrum opportunities for SUs and 
check the state of a channel. Experimental results show the performance gain achieved by SUs with less probabilities of misdetections and false alarm.

UWB (ultra wide band) technology has gained attention recently due to its significance in short range communications. Keeping in view the pros of UWB technology, the authors of [72] present a handoff strategy for cognitive ultra-wide band industrial networks (CUWBINs), where an SU can coexist with a PU on a channel. The busy and idle periods of a channel are modeled by developing Markov chains. This strategy avoids the collisions between SUs and its dynamic and diverse nature provides seamless connectivity to users. Through simulations, it is shown that the proposed proactive handoff models achieve less handoff delay as well as increased handoff efficiency compared to existing schemes which do not utilize UWB in their design process. However, as stated in [92], the coexistence of SUs with PUs results in lower throughput for users.

In [15], the authors propose a 2-state CTMC (continuous time Markov chain) to model the channel availability for SUs by taking in account the secondary user's mobility. A concept known as "guard distance" is introduced which is basically an additional separation between primary and secondary users. The purpose of guard distance is to prevent interference on PU transmissions. This guard distance is then optimized with the "sensing time" to maximize the opportunities in spectrum reuse. Likewise in [107], Markov models are presented with focus on calculating the forced termination and blocking probabilities in scenarios when an SU may or may not perform a handoff. Another related survey having details of Markov and similar prediction schemes can be found in [105].

An approach allowing SUs to opportunistically operate on various vacant PU channels is presented in [114]. The algorithm allows an SU to predict the channel status and decide to stay idle on the current channel or perform handoff, respectively. The whole channel access and handoff scenario is modeled using a discrete time Markov chain that minimizes the total cost of an SU for a specific transmission. Moreover, the proposed strategy achieves a better data transmission efficiency and energy consumption as compared to always staying and always changing handoff schemes [30].

\section{Modified Optimal Target Channel Sequence Design (MOTCSD)}

Wang et al., presented an interesting greedy algorithm in [56] which is based on proactive selection of target channels. Based on network status, the algorithm allows SUs to select suboptimal channels by comparing six different mathematically generated sequences (for details please refer to Theorem 2 and Figure 5 of [56]). The authors of [108], on the other hand, believe that instead of six sequences discussed in [56], a set of five target channel sequences are enough for the production of suboptimal greedy behavior (one of the six sequences is "redundant"). This modification can minimize the processing time spent during optimal target channel selection especially where the candidate channels are huge in numbers as reflected in presented results. Moreover, the algorithm of [108] can sufficiently decrease the probability of selecting the worst channel which was not addressed in [56].

\section{Other Proactive Approaches}

Beside above, there are some other important strategies which are based on proactively sensing the channels and taking handoff decisions. In [40], an efficient spectrum access policy is developed which allows SUs to create a random order for the available channels (before PUs arrival/return), and then find optimal transmission and handoff opportunities in a distributed manner. In [17][111], the authors model the spectrum handoff process as a continuous time Markov chain with a focus on achieving minimal handoff delay. In [70], SUs are allowed to cooperate at several rendezvous points to share the information about PUs activity. All this activity is done proactively before the arrival of PUs. Multiple rendezvous points allow flexibility in SUs cooperation and thus the proposed approach achieves higher throughput than single rendezvous schemes discussed in [70]. Another interesting proactive handoff scheme is proposed in [1]. The authors introduce a novel concept of a channel assigning agent (CAA) under LTE (long term evolution)/WLAN cognitive radio network. The CAA is responsible for allocating channels to CR users as well as handling their movements from one cell to another. Unlike other proactive approaches, the inclusion of CAA in [1] simplifies the handoff management process since the duties of user registration, channel information storage, and 
seamless handoff management are performed by CAA. This performance gain is shown through experimental results in the form of reduced number of handoffs.

\section{Reactive Handoff Schemes}

In reactive handoff strategies, the future channel is selected by instant sensing after the occurrence of handoff triggering event. An SU can continue its paused transmission on newly searched channel [85]. The whole reactive process can be seen in Figure 7 where an SU senses and then moves to channel 2 at PU arrival. The advantage of reactive handoff is the accuracy in selecting the target channel, but as the selection is made from the results of ondemand wideband sensing therefore, reactive approaches mostly suffer with an additional cost of sensing time [64]. Moreover, the handoff delay also increases because the sensing is started after the handoff triggering event. We detail existing reactive solutions as follows.

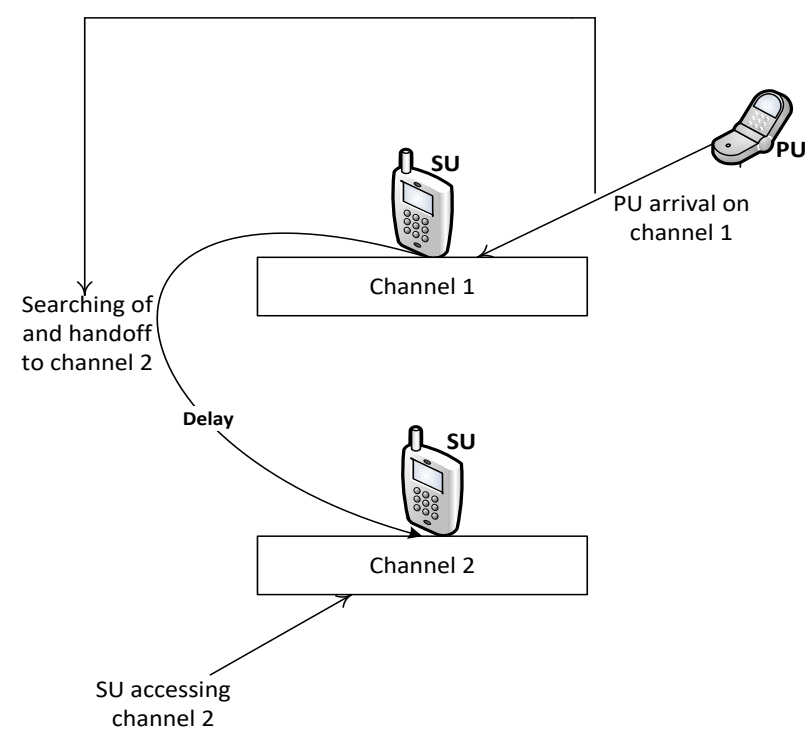

Figure 7. Reactive handoff process from channel 1 to channel 2. Different type of devices are used to differentiate between an SU and a PU

I. Coordination and M/G/1 Queuing Models for Reactive Handoff

The authors of [98] proposed a reactive handoff scheme named as dynamic frequency hopping communities (DFHC). This strategy is supposed to increase IEEE-802.22 performance in terms of QoS requirements of SU while providing reliable and timely spectrum sensing for guaranteeing the PU protection. A WRAN (wireless regional area network) cell (or an SU) while communicating on current channel observes availability of the next target channel. A coordination mechanism is also proposed in this scheme to avoid interference among SUs during sensing phase. Generally, a community leader manages the set of $n$ coordinated SUs in DFHC. The SUs interchange sensing information by the coexistence window which is available at the end of a MAC (medium access control) frame. Another coordination based scheme is presented in [77] for Zigbee CRNs in which an SU can access multiple channels via periodic sensing and spectrum handoff. The authors divide their work in two sections; the monitoring section to obtain the received signal strength and the controlling section to evaluate the obtained result to declare SU presence or absence. For sensing process, an energy detection algorithm [84] is used that compares the received signal's energy with a properly set threshold. If energy is more than the threshold, then the arrival of PU is declared. The handoff is performed by comparing the energy level of a channel with defined threshold. 
Like coordination, developing queuing models is also considered to be an efficient way for handoff management. Thus, in [27][28] $]^{1}[89] \mathrm{M} / \mathrm{G} / 1$ queuing models are proposed to improve the channel usage of SUs. Each SU can simultaneously use multiple available channels for its transmission. Since, the availability of channels directly depends on traffic patterns of a PU, therefore service time is distributed non-trivially. M/G/1 queues are used to model the aggregated arrivals of all SUs sharing the available channels. Further, the proposed model in [27] considers two situations; 1) when the number of SUs accessing the channels is greater than the available channels, then some users must wait for the channel to become free and 2) when the number of SUs is less than the available channels, the packets waiting time remains minimal. The authors of [89], on the other hand, focus on minimizing the overall system time for SUs. This time is defined as a sum of transmission time and waiting time. Through simulations, the authors prove the reduction in overall system time as well as consistency between the results obtained via probabilistic M/G/1 model and experiments. Another priority M/G/1 queuing based reactive handoff scheme is proposed in [26]. In the considered scenario, the PUs and SUs transmit on the same channel simultaneously until and unless an SU causes interference to a PU, resulting in handoff for the corresponding SU. The channel usage patterns and behaviors of primary and secondary users with multiple spectrum handoffs are categorized using M/G/1 queuing. The authors of [76] focus on the aspect of cross layer optimization in order to search for an alternate channel at PU arrival. M/G/1 modeling is used to analyze the transmission performance of SUs for Physical and MAC layers on an aggregate basis.

Both coordination and $\mathrm{M} / \mathrm{G} / 1$ strategies maximize channel usage and user throughput by deploying efficient coordination and queuing mechanisms, however, handoff delay and waiting time for SUs are increased as the handoff decision is taken after the PU arrival. Moreover, the solution of [77] provides better results when the noise is at minimal level, but as the energy detector cannot distinguish between a PU's signal and noise, therefore, a false alarm can be triggered causing unnecessary handoffs. Under multi-path fading or shadowing, a CR user requires higher detection capabilities to tackle with the channel randomness which can be provided by cooperation mechanisms [31][67].

\section{Other Important Reactive Handoff Schemes}

In [33], an interesting reactive scheme is proposed with the focus on throughput maximization of SUs. An SU holds multiple available channels simultaneously and instead of utilizing a whole channel, an SU performs its transmission on multiple sub-channels. When a primary user wants to reclaim all the capacity of its channel, the SU then has to search for another vacant channel to continue its transmission. Through experiments the authors show that their reactive algorithm results in efficient throughput achievement for SUs with a low frequency of handoffs, however, this approach suffers with the complexity of dividing a channel in multiple sub-channels and then avoiding interference to corresponding PUs on each of the sub-channels. Different from [33], a slightly recent work proposed in [104] allows SUs to select future channels (on PU arrival) based on two criteria; the predicted probability of whether the target channel is idle or busy and the length of period for which a channel remains occupied by a PU. If a channel is idle and has shorter busy period, then a handoff is performed on that channel. This decision is then communicated to neighboring SUs. The proposed reactive scheme sufficiently reduces the number of handoffs, however, it requires efficient sensing to monitor the time a PU actually stays on a channel since the handoff decision is totally based on the length of a busy period. Moreover, the communication exchange between SUs also causes an extra overhead.

The authors in [71] propose an interesting reactive handoff approach by introducing realistic channel switching capabilities, where the SUs can only switch to neighboring channels without inducing high latencies. Here, by realistic it means that SUs can only switch to those neighboring channels which are within their frequency range. This is unlikely to happen in other reactive approaches where any channel can be selected at PU arrival without considering device's operating frequency. CTMC models are used to derive the forced termination and blocking probabilities for CR users. Through results, it is shown that increasing PU arrival rate has a direct impact on blocking and forced termination probabilities which is (kind of) an obvious trend.

\footnotetext{
${ }^{1}$ Details about this work are thoroughly discussed in [53].
} 
Normally, most of the above reactive solutions are developed for situations where an SU's transmission is interrupted by a PU only once, thus ignoring the case of multiple PU interruptions. This important concern is addressed in [96] where a delay sensitive reactive strategy is presented for the SUs having multiple interruptions by neighboring PUs. An SU is allowed to access a channel more than once in different time slots. Moreover, an SU should complete its multiple transmissions within a specified time delay which is unlikely to happen in most of the above presented reactive approaches. Through mathematical analysis, the authors calculate buffer size, capacity of a fading channel, average delay in transmission, spectrum access of an SU, and waiting time. Experimental results show that the proposed approach incurs less delay especially for SUs having multiple interruptions.

\section{Hybrid/Adaptive Handoff Schemes}

The schemes we discussed so far are either proactive or reactive in nature. One important aspect recent researchers have focused on, is to combine the benefits of both proactive and reactive handoff schemes to develop an efficient hybrid/adaptive solution. Both words "hybrid" and "adaptive" can be used interchangeably, however, as discussed in our recent work [94], we differentiate between the two terms for the sake of explanation and classification. In a hybrid approach (shown in Figure 8), sensing of a primary user's signals and handoff decision are both performed jointly in proactive and reactive manners, respectively. By continuous sensing, the target channel is selected proactively and the handoff is performed at the occurrence of triggering event, thus shifting to a (proactively) selected channel reactively on the arrival of a PU. In an adaptive solution (shown in Figure 9), "the decisions of channel selection and handoff are made by continuously monitoring the arrival and departure patterns of a PU. When a PU is moving quite regularly, an SU may adapt to reactive handoff strategy. While in case of rare PU movements, a proactive handoff solution is preferred by the corresponding $S U$. Thus, a secondary user can either choose between proactive or reactive approach based on the frequency of PU arrival and departure [94]." Below we detail various hybrid and adaptive handoff schemes.

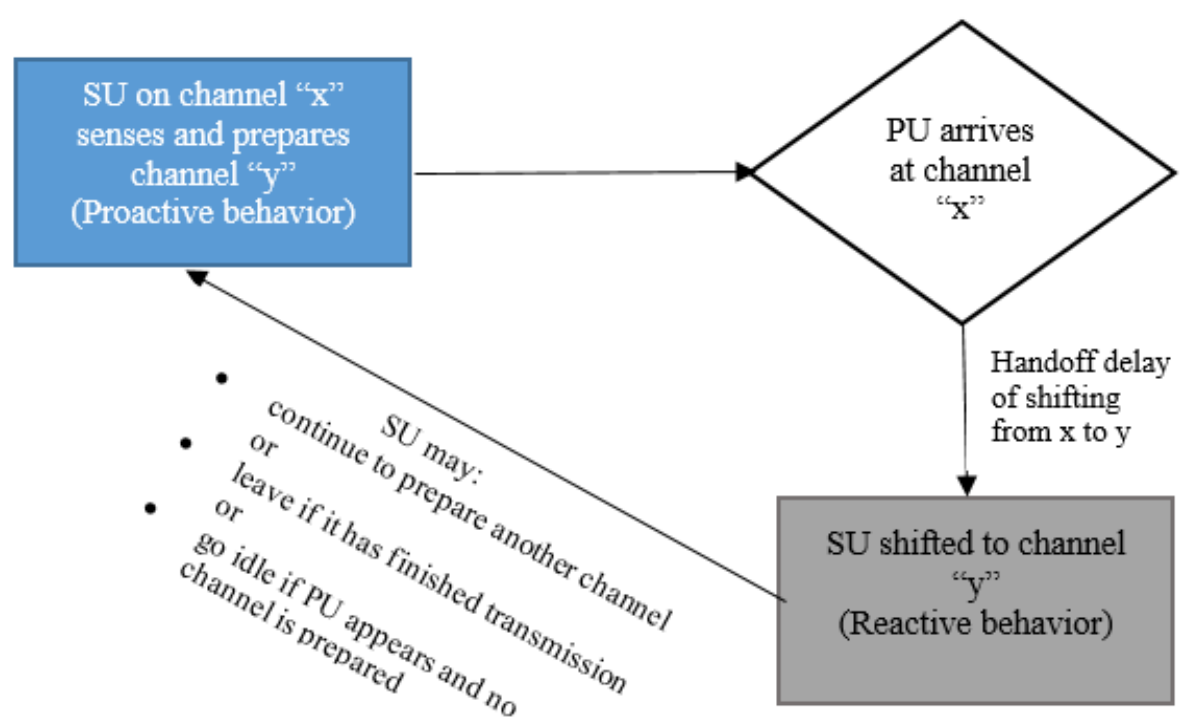

Figure 8. A simple example showing an SU performing hybrid handoff decisions. For simplicity two channels $\mathrm{x}$ and $\mathrm{y}$ are considered

\section{Hybrid Solutions}

Spectrum aggregation (SA) allows a PU or an SU to simultaneously utilize multiple spectrum bands in order to satisfy increased bandwidth demand and achieve better performance in terms of QoS [100]. CR being an intelligent radio can sense and utilize the available white spaces by examining the radio environment [119]. This sensing quality makes it possible to combine the idle channels by spectrum aggregation. A hybrid handoff scheme is proposed in [29], 
which is based on dynamic spectrum aggregation to identify the behavior of an SU during a handoff process. Whenever PU comes back to its licensed channel, the SU performs handoff to any other backup channel. All the channels other than the current channel are backup channels for an SU which can be either idle or utilized by a PU. An SU will wait for the completion of PU transmission if a channel is found busy. An SU should choose minimum handoff probability to reduce the number of handoffs. To minimize handoff delays, backup channels can be used, however, as previously mentioned, this might result in "channel underutilization."

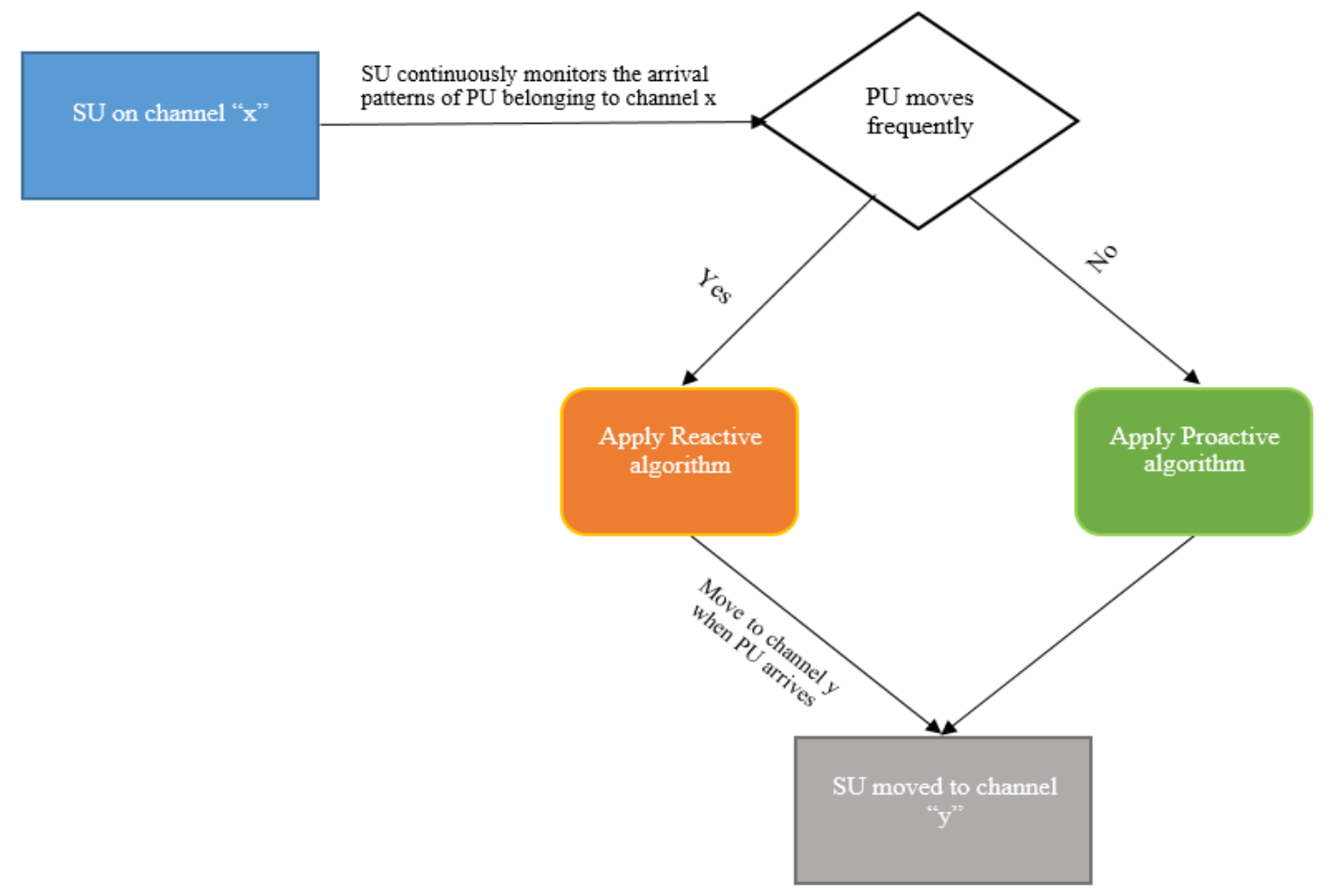

Figure 9. An adaptive handoff strategy

As presented in [29], the hybrid scheme proposed in [80] allows SUs to take a proactive or a reactive handoff decision on the basis of PU arrival rate as well as network parameters such as QoS and bandwidth. The combination of these parameter values along with PU arrival rate on current channel are compared with those of the neighboring sensed channel(s). Handoff decision is performed if the future channel provides higher QoS and bandwidth to an SU. Simulation results show that the proposed hybrid handoff scheme reduces the blocking probability and handoff delay for real-time services; however, it increases the waiting time and blocking probability for non-real time services since SUs have to wait quite a lot to get access to a channel.

The authors of [112] presented a hybrid handoff scheme to maximize the QoS experience of multimedia centric SUs. An SU adaptively senses and selects the target channel before PU arrival and performs the handoff action after the triggering event depending on the varying channel conditions and QoS requirements. A mixed preemptive and non-preemptive resume priority (PRP and NPRP) queuing model is developed to provide differentiated multimedia services to SUs. The PRP captures the interactions between PUs and SUs, while the NPRP queuing is used to depict the behavior of an SU. The proposed queuing model allows the (so called) high priority SUs to continue their transmission without being interrupted by the neighboring SUs, thus achieving high QoS for users running multimedia based applications. Nevertheless, sometimes SUs with no multimedia services have to stay in a waiting queue for long durations, which increases the overall waiting time. Different from [112], the algorithm presented in [20] combines the analytical hierarchy process (AHP) [22] and technique for order of preference by similarity to ideal solution (TOPSIS) [21] to perform a handoff decision. AHP evaluates the decision criteria for the selection of best target channel while TOPSIS evaluates all available channels and arrange them from best to worst. Best channel for handoff 
is selected on the basis of channel availability probability, estimated duration of availability, available bandwidth, and SNR (signal to noise) ratio. Despite being an interesting hybrid algorithm, the proposed simulations are vague and presented results have not been justified properly. Another hybrid scheme is presented in [75] exploiting the benefits of both proactive and reactive decisions, however, this approach suffers with the traditional drawback of large number of handoffs at PU arrival.

\section{Adaptive Solutions}

Unlike reactive, proactive, and hybrid solutions, adaptive handoff approaches are rare to find in the existing literature. In this context, an adaptive handoff strategy is presented in [8] which combines both static and dynamic spectrum access features. In static access, the handoff action is not performed during the transmission of an SU. The channel is assigned only once at the start of transmission. Whereas in dynamic access, an SU can change the operating channel on the arrival of a PU to complete its transmission, thus trying to achieve the objective of maximal channel utilization [101]. Based on the network configuration and PU activity modeling (via heuristics), an SU can shift between static and dynamic modes, respectively. Thus, adapting to a static mode when the channels are idle and shifting to a dynamic mode when PU arrivals are frequent. Slightly different from [8], in [23] the available channels are detected proactively via periodic sensing. After channel selection, the periodic sensing is stopped and whole available time is dedicated to the SUs transmissions. On PU arrival, the transmission of an SU is shifted to the next selected channel. If next channel is busy, then on-demand sensing (as in reactive handoff) is performed to look for another vacant channel, thus, adaptively adjusting to various sensing and handoff modes according to PU activities.

The above two strategies suffer with the traditional limitations of large waiting time, poor channel utilization, and unproductive handoffs. We tried to address these limitations in our recent work published in [94]. We apply energy detection sensing [84] to sense PU arrivals (this type of sensing is less complex in implementation [13]). In case of performing a handoff, the data delivery time [87] for proactive and reactive handoff decisions is calculated and the scheme with minimum data delivery time is applied for proper handoff function. This important factor was ignored in [8] and [23]. Another promising feature of our strategy is to model the overall handoff process using CTMCs [19] which can capture the system evolution dynamics, especially the effects of PU arrivals on SU services. It is shown through extensive simulations that the proposed continuous time models and adaptive nature of algorithm based on data delivery time allow SUs to achieve high spectrum usage, less unproductive handoffs, and minimum waiting delays compared to various existing solutions mentioned above.

Beside all the schemes discussed so far, there are some important ones which are worth mentioning here, however, we are not detailing these strategies because they do not fit into the category of "time triggered handoff." These important works are shown in Figure 10 and are a combination of static and dynamic spectrum sharing, fixed and probabilistic sequences, and preemptive and non-preemptive resume priority, respectively.

\section{PERFORMANCE CRITERIA FOR HANDOFF STRATEGIES}

In this section, we briefly discuss the important performance factors (or criteria) which can seriously affect the performance of a handoff strategy in CRNs. These criteria are chosen by thoroughly examining the existing literature. For the scope of this work, we mostly focus on "time triggering" strategies, however, these criteria are equally important and addressed in all other handoff management approaches.

\section{A. Number of Handoffs}

Spectrum handoff occurs very frequently in CRNs depending on the patterns of primary users. These handoffs can sufficiently decrease the performance of an SU by adding an additional delay and latency in transmission. This may seriously degrade the battery life and energy of an SU device. According to [88], on average 96\% of device's energy is consumed in a single spectrum handoff process [88]. Therefore, decreasing the number of handoffs is an important performance criterion to consider in design of handoff strategies. The schemes reported in [29][91][30][27][78][91][94][17][98][104][112] thoroughly focus on minimizing the number of handoffs in CRNs. 


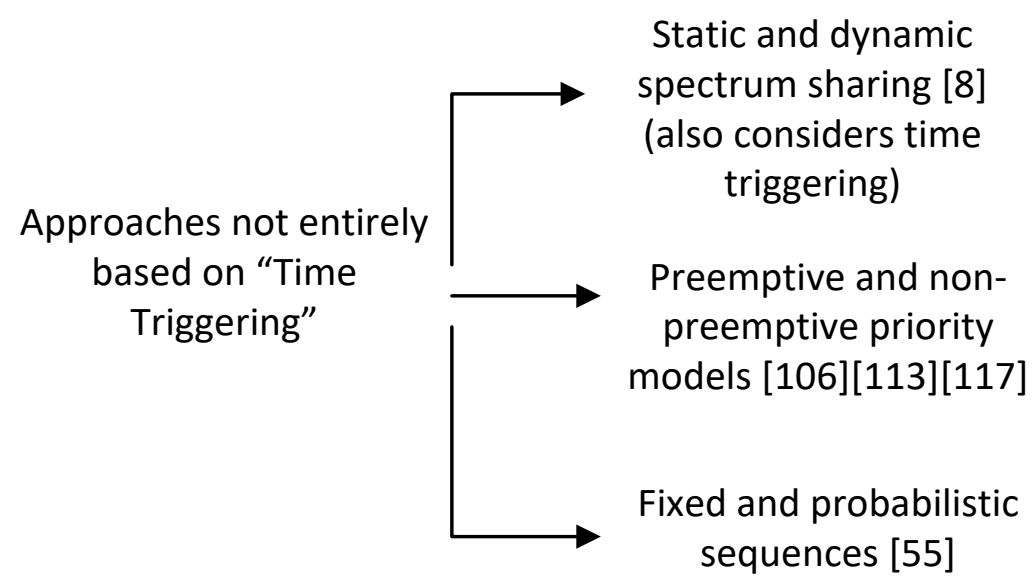

Figure 10. Approaches based on other factors than "time triggering"

\section{B. Channel Utilization}

Bandwidth is a limited and important resource in wireless communications. As discussed several times, the purpose of a CR user is to efficiently utilize the available channels to mitigate the effects of overall spectrum scarcity. Thus, efficient channel utilization is an important design and implementation factor for CRNs and this importance becomes more critical in highly frequent handoff conditions. Spectrum handoff strategies have to be designed without ignoring the overall channel utilization. Almost all the works discussed so far consider channel utilization as a part of their design and implementation process.

\section{Handoff Delay}

Handoff delay is the time an SU takes to pause an ongoing transmission on arrival of a PU until the communication is resumed on the next available channel. Handoff delay of a CR user comprises of waiting time, spectrum sensing time, and channel switching time [57][116]. Increase in handoff delay can cause sufficient degradation in transmission of an SU. Therefore, a good handoff strategy should encounter a minimum handoff delay. The work proposed in [1][8][29][30][72][8][80][20] address handoff delay in detail.

\section{Energy Efficiency}

Energy efficiency is highly desirable in CRNs due to limited lifetime of battery resources. Energy efficiency can sufficiently extend the life of a network. In certain situations such as forests fire detection [68], battle field communications [82], underground mines monitoring [69], disaster management [73], smart grids [2], and high alert zones [81], it is undesirable to replace the battery source of a CR device. The importance of energy becomes even more significant in situations where a user has to switch its on-going transmission to another vacant channel. This involves use of energy during the sensing process (before performing the handoff action). The authors of [41][42] [74][77][87][79] focus on designing energy efficient spectrum handoff algorithms. In addition, the survey presented in [115] highlights important aspects on how to control the transmission power of CR users to save as much energy as possible. 
Table 5. Time triggered handoff schemes with their performance criteria

\begin{tabular}{|c|c|c|c|c|c|c|c|}
\hline Ref. \# & $\begin{array}{c}\text { Number } \\
\text { of } \\
\text { handoffs }\end{array}$ & $\begin{array}{c}\text { Channel } \\
\text { utilization }\end{array}$ & $\begin{array}{c}\text { Delay/Handoff } \\
\text { delay }\end{array}$ & $\begin{array}{c}\text { Energy } \\
\text { efficiency }\end{array}$ & $\begin{array}{c}\text { QoS } \\
\text { assurance }\end{array}$ & $\begin{array}{c}\text { Throughput } \\
\text { maximization }\end{array}$ & $\begin{array}{c}\text { Total } \\
\text { service } \\
\text { time }\end{array}$ \\
\hline [1] & $\checkmark$ & & $\checkmark$ & & & $\checkmark$ & $\checkmark$ \\
\hline [10] & & & $\checkmark$ & & $\checkmark$ & & \\
\hline$[104]$ & $\checkmark$ & $\checkmark$ & & & & & \\
\hline$[107]$ & & & & & & $\checkmark$ & $\checkmark$ \\
\hline [108] & & & & & & & $\checkmark$ \\
\hline [11] & $\checkmark$ & & & & $\checkmark$ & $\checkmark$ & \\
\hline [111] & & & & & & $\checkmark$ & \\
\hline [112] & $\checkmark$ & & & & $\checkmark$ & $\checkmark$ & $\checkmark$ \\
\hline [114] & & $\checkmark$ & & & & & \\
\hline$[12]$ & & & & $\checkmark$ & & & $\checkmark$ \\
\hline [15] & & $\checkmark$ & & & & & $\checkmark$ \\
\hline [17] & $\checkmark$ & $\checkmark$ & $\checkmark$ & & & & \\
\hline [20] & & $\checkmark$ & $\checkmark$ & & & $\checkmark$ & \\
\hline [23] & & $\checkmark$ & & & & & \\
\hline [24] & & $\checkmark$ & & & & & \\
\hline [26] & $\checkmark$ & $\checkmark$ & & & & & \\
\hline [27] & $\checkmark$ & & & & & & $\checkmark$ \\
\hline [28] & & & $\checkmark$ & & & & \\
\hline [29] & $\checkmark$ & & $\checkmark$ & & $\checkmark$ & & \\
\hline [30] & $\checkmark$ & & $\checkmark$ & & & & \\
\hline$[40]$ & & & & & & $\checkmark$ & $\checkmark$ \\
\hline [33] & $\checkmark$ & & & & & $\checkmark$ & \\
\hline [58] & & $\checkmark$ & & & $\checkmark$ & & \\
\hline [63] & & & & & $\checkmark$ & & $\checkmark$ \\
\hline [7] & & & & & & & $\checkmark$ \\
\hline [70] & & & & & & $\checkmark$ & $\checkmark$ \\
\hline [71] & & $\checkmark$ & & & & & \\
\hline [72] & & $\checkmark$ & $\checkmark$ & & & & \\
\hline [74] & & & & & & $\checkmark$ & \\
\hline [76] & & & & & $\checkmark$ & $\checkmark$ & $\checkmark$ \\
\hline [77] & $\checkmark$ & & & $\checkmark$ & & & \\
\hline [78] & $\checkmark$ & & & & & $\checkmark$ & $\checkmark$ \\
\hline [79] & & & & $\checkmark$ & & & \\
\hline [8] & $\checkmark$ & & $\checkmark$ & & & $\checkmark$ & \\
\hline [80] & & & $\checkmark$ & & $\checkmark$ & & \\
\hline [89] & & & & & & & $\checkmark$ \\
\hline [9] & & & $\checkmark$ & & & & \\
\hline [91] & $\checkmark$ & & & & & & \\
\hline [94] & $\checkmark$ & $\checkmark$ & $\checkmark$ & & & $\checkmark$ & $\checkmark$ \\
\hline [96] & & $\checkmark$ & $\checkmark$ & & & & $\checkmark$ \\
\hline [98] & $\checkmark$ & & & & & $\checkmark$ & \\
\hline
\end{tabular}




\section{E. QoS Assurance}

Some applications such as live streaming, video on-demand ( $\mathrm{VoD})$, and online gaming desire for strict QoS requirements in traditional wireless and CR networks, therefore the design of a good handoff strategy should account for these requirements. QoS in CRNs depends on many factors such as transmission rate, PU arrival rate, number of handoffs, handoff delay, waiting time, and probability of false alarm. The handoff schemes proposed in [29][58][76][80][112] consider QoS as an essential part of their design process, however, most of these strategies suffer with the traditional limitations of not achieving maximum channel utilization and energy efficiency [4].

\section{F. Throughout}

Throughput is the total amount of data transferred by an SU in a specific time unit. In CRNs, it can be termed as the throughput achieved by an individual user or the overall throughput of the network. The works proposed in[1][8][20][27][78][98][112] focus on maximizing throughout for secondary users, however, some of the aforementioned also suggest to increase the transmission power for an SU to achieve a maximum throughput value. This may result in harmful interference to the transmission of neighboring PUs. Moreover, number of handoffs affects the overall throughout for SUs since the SUs have to stop their transmission on PU arrival and look for other channels. In some situations, an SU has to remain idle for a long period of time unless the corresponding PU leaves the channel or an SU finds another vacant space to continue transmission. This waiting time results in serious decrement in an SU's throughout.

\section{G. Total Service Time for Secondary Users}

Total service time for an SU is the time it takes to complete the transmission on an allocated channel. Total service time comprises of waiting time, sensing time, channel processing time, and transmission time. The approaches presented in [27][108] consider waiting time to be an important factor affecting the service time of SUs. Likewise, waiting time reduction is shown in the results presented in [78][76]. The authors of [1][7][15][40][58][78][112] also focus on increasing service time of SUs without degrading the performance of PUs.

To provide a more comprehensive analysis we summarize all the time triggered strategies with their performance criteria in Table 5.

\section{CHALLENGES, ISSUES AND FUTURE DIRECTIONS FOR A TIME TRIGGERED HANDOFF PROCESS}

In this section, we discuss the challenges and issues related to a time triggered handoff process and outline the future research directions and opportunities. Without the loss of generality, these issues are equally important for all sorts of handoff schemes.

\section{A. Intelligent Handoff Process}

Most of the existing time triggered handoff schemes lack the spectrum learning feature in their design [46]. Ideally, an SU should know the traffic patterns of a PU via its sensing capabilities and adapt to most suitable handoff technique wherever needed. As soon as the traffic patterns of a PU change, the SU should notice that and choose between, a proactive or a reactive handoff decision, accordingly. Despite the fact that various schemes consider sensing as an important factor in their design, still this issue requires further in depth investigation. In addition, device's energy factor should be kept in consideration while designing a learning-based sensing/handoff strategy. We recommend researchers to go through an important work presented in [62]. Though, this intelligent handoff scheme is designed for virtual wireless networks (addressing a specific train communication scenario), it can easily be adjusted within the context of a CRN. 


\section{B. Priority Based Spectrum Handoff}

In case of an emergency, such as natural or man-made disasters and health care emergencies or accidents, and security related situations, the spectrum of a PU can opportunistically be used by an SU in order to provide communication [32][39]. These emergency conditions require channel selection and handoff to be performed rather quickly compared to normal situations. Thus, a patient in an ambulance would desire for a priority e-health services with better mobility and QoS support compared to another person sitting at home trying to utilize bandwidth for his or her routine stuff. Therefore, spectrum handoff schemes should be designed by keeping in mind the fast varying nature of modern day applications.

\section{Interference Avoidance}

Primary users are the licensed owners of spectrum whereas the SUs have opportunistic access to the spectrum. The simultaneous appearance of an SU on a licensed spectrum band can cause harmful interference to a PU. This is a major issue in CRNs. The interference to the PU's transmission should be avoided during the occurrence of handoff. For an accurate and efficient handoff process, the interference avoidance mechanism should be proposed in design process of a handoff strategy [53]. Moreover, to further mitigate the effects of users' interferences, next channel selection and handoff should be performed with minimal delay. This requires a suitable organization of an SU's traffic in real time [54]. Some recent researchers also suggest that it is rather useful to consider the interference between users as an important resource especially when traditional wireless and CR users coexist. Interested readers may refer to a promising recent work presented in [44].

\section{Optimization of Parameters Involved in Handoff Decision}

It is difficult and time consuming to consider the impact of all the parameters (such as throughput, number of handoff, etc.) in designing a handoff strategy due to the architectural complexity constraints. Therefore, parameters should be prioritized according to the situation. For example, in an ad-hoc scenario, special attention should be given to the random movements of SUs and PUs which may seriously affect the handoff process. In some other static situations, utility or throughput maximization could be prioritized since the users movements are not random and can be ignored in the design process. Thus, "dynamic adjustment/mining" of all important parameters is an important current and future challenge [60].

\section{CONCLUSION}

In this paper, spectrum handoff approaches based on time triggering are summarized. Various existing schemes are classified in non-proactive, reactive, and hybrid/adaptive handoff categories and their pros and cons are discussed in detail. Several figures and tables are provided to analyze time triggered handoff approaches in a comparative manner. We also discussed several important parameters such as throughput, delay, number of handoffs, and various others which are important in designing a handoff strategy. In future, we will continue to present any further research and advancement related to cognitive radio networks.

\section{REFERENCES}

[1] A. A. Dulaimi, et al., "Spectrum Handoff Management in Cognitive HetNet Systems Overlaid With Femtocells," IEEE Systems Journal, vol. 10, pp. 335-345, 2016.

[2] A. A. Khan, et al., "Cognitive radio for smart grids: Survey of architectures, spectrum sensing mechanisms, and networking protocols," IEEE Communications Surveys \& Tutorials, vol. 18, pp. 860-898, 2016.

[3] A. A. Khan, et al., "Cognitive-Radio-Based Internet of Things: Applications Architectures Spectrum Related Functionalities and Future Research Directions," IEEE Wireless Communications, vol. 24, pp. 17-25, 2017.

[4] A. Ahmad, et al., "A survey on radio resource allocation in cognitive radio sensor networks," IEEE Communications Surveys \& Tutorials, vol. 17, pp. 888-917, 2015.

[5] A. Ali, et al., "Advances on Spectrum Sensing for Cognitive Radio Networks: Theory and Applications," IEEE Communications Surveys and Tutorials, vol. 19, pp. 1277-1304, 2016.

[6] A. F. Tayel, et al., "Load balancing probabilistic spectrum handoff for cognitive radio networks", In International Symposium on Performance Evaluation of Computer and Telecommunication Systems (SPECTS), pp. 1-8, 2015. 
[7] A. F. Tayel, et al., "An optimized hybrid approach for spectrum handoff in cognitive radio networks with non-identical channels," IEEE Transactions on Communications, vol. PP, pp. 1-10, 2016.

[8] A. Lertsinsrubtavee, et al., "Hybrid spectrum sharing through adaptive spectrum handoff for cognitive radio networks." In IFIP Networking Conference, pp. 1-9, 2014.

[9] A. Lertsinsrubtavee, et al., "Spectrum handoff strategies for multiple channels cognitive radio network," In ACM CoNEXT Student Workshop, pp. 20-21, 2010.

[10] A. Lertsinsrubtavee, et al., "Spectrum handoff strategy using cumulative probability in cognitive radio networks," In International Congress on Ultra Modern Telecommunications and Control Systems and Workshops (ICUMT), pp. 1-7, 2011.

[11] A. Lertsinsrubtavee, et al., "Hybrid spectrum sharing through adaptive spectrum handoff and selection," IEEE Transactions on Mobile Computing, pp. 1-13, 2016.

[12] A. O. Bicen, et al., "Dedicated Radio Utilization for Spectrum Handoff and Efficiency in Cognitive Radio Networks," IEEE Transactions on Wireless Communications, vol. 14, pp. 5251-5259, 2015.

[13] A. S. Cacciapuoti, et al., "Channel availability for mobile cognitive radio networks," Elsevier Journal of Network and Computer Applications, vol. 47, 131-136, 2015.

[14] A. Tonmukayakul, and M. B. H. Weiss, “An agent-based model for secondary use of radio spectrum,” In IEEE DySPAN, pp. 467-475, 2005.

[15] A. W. Min, et al., "Opportunistic spectrum access for mobile cognitive radios," In IEEE INFOCOM, pp. 2993-3001, 2011.

[16] B. Benmammar, et al., "A Survey on Dynamic Spectrum Access Techniques in Cognitive Radio Networks," International Journal of Communication Networks and Information Security, vol. 5, pp. 68-79, 2013.

[17] B. Kahraman, et al., "An efficient and adaptive channel handover procedure for cognitive radio networks," Wireless Communications and Mobile Computing, vol. 15, pp. 442-458, 2015.

[18] B. Wang, et al., "Advances in cognitive radio networks: A survey," IEEE Journal of selected topics in signal processing, vol. 5, pp. 5-23, 2011.

[19] B. Wang, et al., "Primary-prioritized Markov approach for dynamic spectrum access," IEEE Transactions on Wireless Communications, vol. 8, pp. 1854-1865, 2009.

[20] C. Hernández, et al., "Hybrid Algorithm for Frequency Channel Selection in Wi-Fi Networks," World Academy of Science, Engineering and Technology, vol. 9, pp. 80-83, 2015.

[21] C. Hernandez, et al., "MCDM Spectrum Handover Models for Cognitive Wireless Networks," World Academy of Science, Engineering and Technology, vol. 9, pp. 679-682, 2015.

[22] C. Hernandez, et al., "Multivariable algorithm for dynamic channel selection in cognitive radio networks," EURASIP Journal of Wireless Communications and Networking, vol. 2015, pp. 1-17, 2015.

[23] C. Pham, and H. S. Chong, "Adaptive Sensing Scheduling with Hybrid Sensing Model," In International Conference on Computer Science and Information, pp. 1008-1010, 2013.

[24] C. Pham, et al., "Spectrum handoff model based on Hidden Markov model in Cognitive Radio Networks." In IEEE International Conference on Information Networking (ICOIN), pp. 406-411, 2014.

[25] C. Q Luo, et al., "Joint relay scheduling, channel access, and power allocation for green cognitive radio communications," IEEE Journal on Selected Areas in Communications, vol. 33, pp. 922-932, 2015.

[26] C. W. Wang, et al., "Performance gains for spectrum utilization in cognitive radio networks with spectrum handoff," In International Symposium on Wireless Personal Multimedia Communications (WPMC), 2009.

[27] C. W. Wang, et al., "Analysis of Reactive Spectrum Handoff in Cognitive Radio Networks," IEEE Journal on Selected Areas in Communication, vol. 30, pp. 2016-2028, 2012.

[28] C. W. Wang, et al., "Modeling and Analysis for Reactive-decision Spectrum Handoff in Cognitive Radio Networks," In IEEE GLOBECOM, 2010.

[29] C. Yin, et al., "A Hybrid Handoff Strategy Based on Dynamic Spectrum Aggregation in Cognitive Radio System,” In IEEE TENCON Spring Conference, pp. 213 - 217, 2013.

[30] C-W. Wang et al., "Modeling and Analysis for Proactive-decision Spectrum Handoff in Cognitive Radio Networks," In IEEE ICC, 2009.

[31] D. Cabric, et al., "Implementation Issues in Spectrum Sensing for Cognitive Radios," In IEEE Asilomar Conference on Signals, Systems and Computers, pp. 772-776, 2004.

[32] D. Maldonado, et al., "Cognitive radio applications to dynamic spectrum allocation: a discussion and an illustrative example," In IEEE DySPAN, pp. 597-600, 2005.

[33] D. Willkomm, et al., "Reliable link maintenance in cognitive radio systems," In IEEE DySPAN, pp. 371-378, 2005.

[34] E. Z. Tragos, et al., "Spectrum assignment in cognitive radio networks: A comprehensive survey," IEEE Communications Surveys \& Tutorials, vol. 15, pp. 1108-1135, 2013.

[35] F. Sheikholeslami et al., "Optimal probabilistic initial and target channel selection for spectrum handoff in cognitive radio networks," IEEE Transactions on Wireless Communication, vol. 14, pp. 570-84, 2015.

[36] FCC, ET Docket No 03-322 Notice of Proposed Rule Making and Order, 2003.

[37] G. Ghosh, et al., "Cognitive Radio and Dynamic Spectrum Access-A Study," International Journal of Next-Generation Networks, vol. 6, pp. 43-60, 2014.

[38] G. J. Clark, et al., "Architecture for Cognitive Networking within NASA's Future Space Communications Infrastructure," In 34th AIAA International Communications Satellite Systems Conference, International Communications Satellite Systems Conferences (ICSSC), 2016. https://doi.org/10.2514/6.2016-5725 
[39] G. Rathee, et al., "Aspects of secure communication during spectrum handoff in cognitive radio networks.” In International Conference on Signal Processing and Communication (ICSC), December 2016.

[40] H. Shokri and C. Fischione, "Analysis and optimization of random sensing order in cognitive radio networks," IEEE Journal on Selected Areas in Communications, vol. 33, pp. 803-819, 2015.

[41] H. Shokri, et al., "Green sensing and access: energy-throughput trade-offs in cognitive networking." IEEE Communications Magazine, vol. 53, pp. 199-207, 2015.

[42] H. Shokri, et al., "Energy efficient spectrum sensing and handoff strategies in cognitive radio networks," arXiv preprint arXiv:1312.0045, 2013.

[43] H. Sun, et al., "Wideband spectrum sensing for cognitive radio networks: a survey." IEEE Wireless Communications, vol. 20, pp. 74-81, 2013.

[44] H. Zhang et al., "Cooperative Precoding for Wireless Energy Transfer and Secure Cognitive Radio Coexistence Systems," IEEE Signal Processing Letters, vol. 24, pp. 540-544, 2017.

[45] I. Bajaj et al., "Outage-Constrained Sensing Threshold Design for Decentralized Decision-Making in Cognitive Radio Networks", IEEE Transactions on Communications, vol. 64, pp. 4956-4965, 2016.

[46] I. Christian, et al., "Spectrum Mobility in Cognitive Radio Networks," IEEE Communications Magazine, vol. 50, pp. 114$121,2012$.

[47] I. F. Akyildiz, et al. "A survey on spectrum management in cognitive radio networks." IEEE Communications Magazine, vol. 46, no. 4, pp. 40-48, 2008.

[48] I. F. Akyildiz, et al., "Cooperative spectrum sensing in cognitive radio networks: A survey," Physical Communication, vol. 4, pp. 40-62, 2011.

[49] I. F. Akyildiz, et al., "Next Generation/Dynamic Spectrum Access/Cognitive Radio Wireless Networks: A Survey," Elsevier Computer Networks, vol. 50, pp. 2127-2159, 2006.

[50] J. Mitola, "Cognitive radio: An integrated agent architecture for software defined radio," PhD Dissertation, KTH Royal Institute of Technology, Sweden 2000.

[51] J. Mitola, and Jr, G. Q. Maguire, “Cognitive radio: making software radios more personal," IEEE Personal Communications, vol. 6, pp. 13-18, 1999.

[52] J. W. Mwangoka et al., "Cognitive mobility management in heterogeneous networks," In ACM International Workshop on Mobility Management and Wireless Access, pp. 37-44, 2010.

[53] K. Kumar, et al., "Spectrum handoff in cognitive radio networks: A classification and comprehensive survey." Elsevier Journal of Network and Computer Applications, vol. 61, pp. 161-188, 2016.

[54] K. Kumar, et al., "Supporting real-time traffic in cognitive radio networks," In IEEE International Conference on Signal Processing and Communication Engineering Systems, pp. 482 - 485, 2015.

[55] K. Tiwari, et al., "Spectrum Handoff in Cognitive Radio Network." International Journal of Advanced Research in Computer and Communication Engineering, vol. 5, pp.1025-1030, 2016.

[56] L. C. Wang, et al., "Optimal target channel sequence design for multiple spectrum handoffs in cognitive radio networks," IEEE Transactions on Communications, vol. 60, pp. 2444-2455, 2012.

[57] L. C. Wang, et al., "Modeling and Analysis for Spectrum Handoffs in Cognitive Radio Networks," IEEE Transactions on Mobile Computing, vol. 11, pp. 1499-1513, 2011.

[58] L. Giupponi, et al., "Fuzzy-based spectrum handoff in cognitive radio networks," In International Conference on Cognitive Radio Oriented Wireless Networks and Communications (CrownCom), pp. 1-6. 2008.

[59] L. Sun, et al., "The Impact of Network Size and Mobility on Information Delivery in Cognitive Radio Networks," IEEE Transactions on Mobile Computing, vol. 15, pp. 217-231, 2016.

[60] L. Xingya et al., "A Self-Adaptive Optimal Fragmentation Protocol for Multi-Channel Cognitive Radio Ad Hoc Networks," In IEEE GLOBECOM, pp.1-6, 2016.

[61] L. Zhang et al., "Grade of service of opportunistic spectrum access based cognitive cellular networks," IEEE Wireless Communications, vol. 20, pp. 126-smart 33, 2013.

[62] L. Zhu et al., "Handoff Performance Improvements in an Integrated Train-Ground Communication System Based on Wireless Network Virtualization," IEEE Transactions on Intelligent Transportation Systems, vol. 18, pp. 1-14, 2017.

[63] L-C. Wang et al., "Load-Balancing Spectrum Decision for Cognitive Radio Networks," IEEE Journal on Selected Areas in Communications (JSAC), vol. 29, pp. 757-769, 2011.

[64] L-C. Wang et al., and Kai-Ten Feng, "A Queueing-Theoretical Framework for QoS-Enhanced Spectrum Management in Cognitive Radio Networks," IEEE Wireless Communications Magazine, vol. 18, pp. 18-26, 2011.

[65] M. B. Sajjan, et al., "Spectrum Aware Mobility Management in Cognitive Radio-A Survey," International Journal for Innovative Research in Science and Technology, vol. 1, pp. 78-84, 2015.

[66] M. Fahimi, et al., "Joint spectrum load balancing and handoff management in cognitive radio networks: a non-cooperative game approach," Springer Wireless Networks, vol. 22, pp. 1161-1180, 2016.

[67] M. Gandetto, et al., "Spectrum Sensing: a Distributed Approach for Cognitive Terminals," IEEE Journal on Selected Areas in Communication, vol. 25, pp. 546-557, 2007.

[68] M. Hefeeda and M. Bagheri, "Wireless sensor networks for early detection of forest fires," In IEEE MASS, pp. 1-6. 2007.

[69] M. Li and Y. Liu, "Underground coal mine monitoring with wireless sensor networks," ACM Transactions on Sensor Networks, vol. 5, pp. 1-29, 2009. 
[70] M. Mehrnoush, et al., "A distributed spectrum handoff MSRV protocol for the cognitive radio ad hoc networks," Springer Wireless Networks, doi:10.1007/s11276-017-1446-9, 2017.

[71] M. N. Oliaee, et al., "Analyzing cognitive network access efficiency under limited spectrum handoff agility," IEEE Transactions on Vehicular Technology, vol. 63, pp. 1402-1407, 2014.

[72] M. P. Tran, et al., "Effective spectrum handoff for cognitive UWB industrial networks." In IEEE Conference on Emerging Technologies \& Factory Automation (ETFA), pp. 1-4, 2015.

[73] M. S. George et al., "DistressNet: A wireless ad hoc and sensor network architecture for situation management in disaster response," IEEE Communications Magazine, vol. 48, pp. 128-136, 2010.

[74] M. Usman, et al., "Energy-efficient channel handoff for sensor network-assisted cognitive radio network," Sensors, vol. 15, pp. 18012-18039, 2015.

[75] N. A. Lala, et al., "Novel hybrid spectrum handoff for cognitive radio networks," International Journal of Wireless and Microwave Technologies (IJWMT), vol. 3, pp. 1-10, 2013.

[76] N. S. Shankar., "Squeezing the most out of cognitive radio: a joint MAC/PHY perspective," In IEEE International Conference on Acoustics, Speech and Signal Processing (ICASSP), vol. 4, pp. 1361-1364, 2007.

[77] P. Vijayakumar, et al., "Energy Efficient Spectrum Sensing and Accessing Scheme for Zigbee Cognitive Networks," International Journal of Engineering Research and General Science, vol. 10, pp. 128-136, 2014.

[78] P. Yi, et al., “A Novel Spectrum Handoff Method Based On Spectrum Reservation,” TELKOMINIKA Indonesian Journal of Electrical Engineering, Vol.12,pp. 653- 660, 2014.

[79] R. Chai, et al., "Energy efficiency-based joint spectrum handoff and resource allocation algorithm for heterogeneous CRNs," EURASIP Journal on Wireless Communications and Networking, vol. 2016, no. 1, article, 213, 2016.

[80] R. Dahiya, "Combined Algorithm For Minimize the Blocking Probability of Real Time and Non-Real Time Services in Handoff," International Journal of Emerging Trends in Science and Technology, vol. 1, pp. 746-752, 2014.

[81] R. K. Sharma et al., "Advances on security threats and countermeasures for cognitive radio networks: A survey," IEEE Communications Surveys \& Tutorials, vol. 17, pp. 1023-1043, 2015.

[82] R. Murty, "Software-defined re-configurability radios: smart, agile, cognitive, and interoperable" Technology@ Intel Magazine, 2003.

[83] S. Althunibat, et al., "Towards energy-efficient cooperative spectrum sensing for cognitive radio networks: an overview," Telecommunication Systems, vol. 59, pp. 77-91, 2015.

[84] S. Chatterjee, et al., "On optimal threshold selection in cooperative spectrum sensing for cognitive radio networks: An energy detection approach using fuzzy entropy maximization," Springer Wireless Personal Communications, vol. 84, pp. 1605-1625, 2015.

[85] S. Haykin, "Fundamental issues in cognitive radio," Cognitive Wireless Communication Networks, pp. 1-43. Springer US, 2007.

[86] S. Haykin, “Cognitive dynamic systems," IEEE Journal on Selected Areas in Communications, vol. 94, pp. 1910-1911, 2006.

[87] S. Pandya, et al., "Energy detection based spectrum sensing for cognitive radio network," IEEE International Conference on Communication Systems and Network Technologies (CSNT), pp. 201-206, 2015.

[88] S. S. Byun, et al., "Dynamic spectrum allocation in wireless cognitive sensor networks: Improving fairness and energy efficiency,” IEEE VTC, pp. 1-5, 2008.

[89] S. T. Talat, et al., "Spectrum decision for cognitive radio networks with various-bandwidth channels," In IEEE WCNC, 2016.

[90] S. Verma, et al., "A Survey on Spectrum Mobility in Cognitive Radio Network," International Journal of Computer Applications, vol. 119, pp. 33-36, 2015.

[91] S. Zahed, et al., "Analytical modeling for spectrum handoff decision in cognitive radio networks," Elsevier Simulation Modelling Practice and Theory, vol. 38, pp. 98-114, 2013.

[92] T. C. Clancy, et al., "Achievable capacity under the interference temperature model,” In IEEE INFOCOM, pp. 794-802, 2007.

[93] T. Yucek, et al., "A survey of spectrum sensing algorithms for cognitive radio applications," IEEE Communications Surveys \& Tutorials, vol. 11, pp. 116-130, 2009.

[94] U. Mir, and A. Munir, "An Adaptive Handoff Strategy for Cognitive Radio Networks," Springer Wireless Networks (WINE), pp. 1-16, 2017.

[95] U. Mir, L. Merghem-Boulahia, and D. Gaït, "On using Multiagent Systems for Spectrum Sharing in Cognitive Radios Networks," in Proc. Formal and Practical Aspects of Autonomic Computing and Networking: Specification, Development and Verification, IGI Global, pp. 377-417, 2011. DOI: 10.4018/978-1-60960-845-3.ch013, ISBN13: 9781609608453, ISBN10: 1609608453, EISBN13: 9781609608460

[96] U. S. Premarathne, et al., "Reliable Delay-Sensitive Spectrum Handoff Management for Re-entrant Secondary Users," Elsevier Ad Hoc Networks, 2017. https://doi.org/10.1016/j.adhoc.2017.04.008

[97] W. Feng, et al., "Performance evaluation of IEEE 802.19.1 coexistence system,” IEEE ICC, pp. 1-6, 2011.

[98] W. Hu, et al., "Cognitive Radios for Dynamic Spectrum Access - Dynamic Frequency Hopping Communities for Efficient IEEE 802.22 Operation,” IEEE Communications Magazine, vol. 45, pp. 80 -s87, 2007.

[99] W. Hu, et al., "Dynamic Frequency Hopping communities for Efficient IEEE 802.22 Operation," IEEE Communications Magazine, vol. 45, pp. 80-87, 2007.

[100] W. Wang, et al., "Spectrum aggregation: Overview and challenges." Network Protocols and Algorithms, vol. 2, pp. 184196, 2010. 
[101] W. Y. Lee and I. F. Akyldiz, "A spectrum decision framework for cognitive radio networks," IEEE Transactions on Mobile Computing, vol. 10, pp. 161-174, 2011.

[102] W-Y, Lee, et al., "Spectrum-aware mobility management in cognitive radio cellular networks," IEEE Transactions on Mobile Computing, vol. 11, pp. 529-542, 2012.

[103] X. Huang et al., "On green-energy-powered cognitive radio networks." IEEE Communications Surveys \& Tutorials, vol. 17 , pp. 827-842, 2015.

[104] X. Tian, et al., "Second Users Operation Strategies Based on Primary Users Activities," Scientific Journal of Information Engineering, vol. 4, pp. 1-7, 2014.

[105] X. Xing, et al., "Spectrum prediction in cognitive radio networks," IEEE Wireless Communications, vol. 20, pp. 90-96, 2013.

[106] X. Yang et al., "Spectrum Handoffs Based on Preemptive Repeat Priority Queue in Cognitive Radio Networks," Sensors (Basel). 16 (7), 2016, doi: 10.3390/s16071127.

[107] X. Zhu, et al., "Analysis of cognitive radio spectrum access with optimal channel reservation," IEEE Communications Letters, vol. 11, pp. 304-306, 2007.

[108] Y. A. Zakariya, et al., "Comments on "Optimal Target Channel Sequence Design for Multiple Spectrum Handoffs in Cognitive Radio Networks," IEEE Transactions on Communications, vol. 63, pp. 3021-3024, 2015.

[109] Y. Song and J. Xie, "Performance Analysis of Spectrum Handoff for Cognitive Radio Ad Hoc Networks without Common Control Channel under Homogeneous Primary Traffic,” In IEEE INFOCOM, pp. 3011-3019, 2011.

[110] Y. Song and J. Xie, "ProSpect: A proactive spectrum handoff framework for cognitive radio Ad Hoc networks without common control channel," IEEE Transactions on Mobile Computing, vol. 11, pp. 1127-1139, Jul. 2012.

[111] Y. Song, et al., "Common hopping based proactive spectrum handoff in cognitive radio Ad hoc networks," In IEEE GLOBECOM, pp. 1-5, 2010.

[112] Y. Wu, et al., "A learning-based QoE-driven spectrum handoff scheme for multimedia transmissions over cognitive radio networks," IEEE Journal on Selected Areas in Communications, vol. 32, pp. 2134-2148, 2014.

[113] Y. Wu, et al., "Optimal spectrum handoff control for CRN based on hybrid priority queuing and multi-teacher apprentice learning," IEEE Transactions on Vehicular Technology, vol. pp. 1-12, 2016.

[114] Y. Wu, et al., "Delay-constrained optimal transmission with proactive spectrum handoff in cognitive radio networks," IEEE Transactions on Communications, vol. 64, pp. 2767-2779, 2016.

[115] Y. Xu, et al., "Robust power control and beamforming in cognitive radio networks: A survey," IEEE Communications Surveys \& Tutorials, vol. 17, pp. 1834-1857, 2015.

[116] Y. Zhang, "Spectrum handoff in cognitive radio networks: Opportunistic and negotiated situations," In IEEE ICC, pp. 16. 2009.

[117] Z. Y. Ahmed et al., "Analysis of an interruption-based priority for multi-class secondary users in cognitive radio networks," In IEEE ICC, pp. 1-6, 2016.

[118] Y. Saleem and M. H. Rehmani, "Primary Radio User Activity Models for Cognitive Radio Networks: A Survey," Elsevier Journal of Networks and Computer Applications, vol. 43, pp. 1-16, 2014.

[119] F. Akhtar, et al., "White Space: Definitional Perspectives and their Role in Exploiting Spectrum Opportunities," Telecommunications Policy, vol. 40, pp. 319-331, 2016.

[120] L. Yang, et al., "Proactive Channel Access in Dynamic Spectrum Networks," Elsevier Physical Communication, vol. 1, pp. 103-111, 2008.

[121] Y.-C. Liang, et al., "Sensing-throughput tradeoff for cognitive radio networks", IEEE Transactions on Wireless Communications, vol. 7, pp. 1326-1337, 2008. 